\title{
Multiomics-based analyses of KPNA2 highlight its multiple potentials in hepatocellular carcinoma
}

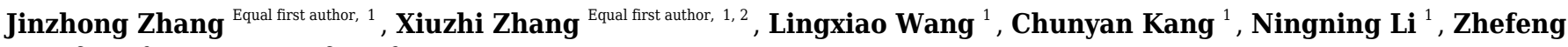 \\ Xiao ${ }^{\text {Corresp., } 2}$, Liping Dai ${ }^{\text {Corresp. } 3}$ \\ ${ }^{1}$ Department of Pathology, Henan Medical College, Zhengzhou, Henan Province, China \\ 2 NHC Key Laboratory of Cancer Proteomics, Xiangya Hospital, Central South University, Changsha, Hunan Province, China \\ 3 Henan Institute of Medical and Pharmaceutical Sciences, Zhengzhou University, Zhengzhou, Henan Province, China \\ Corresponding Authors: Zhefeng Xiao, Liping Dai \\ Email address: xiaozf@csu.edu.cn, Ipdai@zzu.edu.cn
}

Dysregulation and prognostic roles of Karyopherin $\alpha 2$ (KPNA2) were reported in many malignancies including hepatocellular carcinoma (HCC). A multi-omics analysis of KPNA2 is needed to gain a deeper understanding of its multilevel molecular characteristics and provide novel clues for HCC diagnosis, prognosis, and target therapy. Herein, the multiomic alterations of KPNA2 were analyzed at genetic, epigenetic, transcript, and protein levels with evaluation of their relevance with clinicopathological features of HCC by integrative analyses. The significant correlations of KPNA2 expression with its gene copy number variation (CNV) and methylation status were shown through Spearman correlation analyses. With Cox regression, Kaplan-Meier survival, and receiver operating characteristic (ROC) analyses, based on KPNA2 CNV, methylation, expression, and tumor stage, the risk models for HCC overall survival (OS) and disease-free survival (DFS) were constructed which could discriminate the 1-year, 3-year, and 5-year OS/DFS status effectively. With Microenvironment Cell Populations-counter (MCP-counter), the immune infiltrations of HCC samples were evaluated and their associations with KPNA2 were shown. KPNA2 expression in liver was found to be influenced by low fat diet and presented significant correlations with fatty acid metabolism and fatty acid synthase activity in HCC. KPNA2 was detected lowered in HCC patient's plasma by enzyme linked immunosorbent assay (ELISA), consistent with its translocation to nuclei of HCC cells. In conclusion, KPNA2 multilevel dysregulation in $\mathrm{HCC}$ and its correlations with immune infiltration and fatty acid metabolism pathway indicated its multiple roles in HCC. The clinicopathological significance of KPNA2 was highlighted through the in-depth analyses at multilevels. 
1 Multiomics-based Analyses of KPNA2 highlight its multiple

2 potentials in Hepatocellular Carcinoma

3 Jinzhong Zhang ${ }^{1, \dagger}$, Xiuzhi Zhang ${ }^{1,2, \dagger}$, Lingxiao Wang ${ }^{1}$, Chunyan Kang1, Ningning Li ${ }^{1}$,

4 Zhefeng Xiao ${ }^{2,}$, , Liping Dai ${ }^{3, *}$

51 Department of Pathology, Henan Medical College, Zhengzhou, Henan Province, China.

62 NHC Key Laboratory of Cancer Proteomics, Xiangya Hospital, Central South University,

7 Changsha, Hunan Province, China.

83 Henan Institute of Medical and Pharmaceutical Sciences, Zhengzhou University, Zhengzhou, 9 China

$10 \dagger$ These authors contribute equally to this work.

11 * Correspondence:

12 Zhefeng Xiao, xiaozf@csu.edu.cn; Liping Dai, 1pdai@zzu.edu.cn

\section{Abstract}

14 Dysregulation and prognostic roles of Karyopherin $\alpha 2$ (KPNA2) were reported in many malignancies including hepatocellular carcinoma (HCC). A multi-omics analysis of KPNA2 is needed to gain a deeper understanding of its multilevel molecular characteristics and provide novel clues for HCC diagnosis, prognosis, and target therapy. Herein, the multi-omic alterations of KPNA2 were analyzed at genetic, epigenetic, transcript, and protein levels with evaluation of their relevance with clinicopathological features of HCC by integrative analyses. The significant correlations of KPNA2 expression with its gene copy number variation (CNV) and methylation status were shown through Spearman correlation analyses. With Cox regression, Kaplan-Meier survival, and receiver operating characteristic (ROC) analyses, based on KPNA2 CNV, methylation, expression, and tumor stage, the risk models for HCC overall survival (OS) and disease-free survival (DFS) were constructed which could discriminate the 1-year, 3-year, and 5year OS/DFS status effectively. With Microenvironment Cell Populations-counter (MCP-counter), the immune infiltrations of $\mathrm{HCC}$ samples were evaluated and their associations with KPNA2 were shown. KPNA2 expression in liver was found to be influenced by low fat diet and presented significant correlations with fatty acid metabolism and fatty acid synthase activity in HCC. KPNA2 was detected lowered in HCC patient's plasma by enzyme linked immunosorbent assay (ELISA), consistent with its translocation to nuclei of HCC cells. In conclusion, KPNA2 multilevel dysregulation in $\mathrm{HCC}$ and its correlations with immune infiltration and fatty acid metabolism pathway indicated its multiple roles in HCC. The clinicopathological significance of KPNA2 was highlighted through the in-depth analyses at multilevels.

34 Keywords: hepatocellular carcinoma, KPNA2, genetic and epigenetic regulation, prognosis, 35 fatty acid metabolism, immunoregulation. 


\section{INTRODUCTION}

As the most commonly diagnosed malignant tumor in liver with a 5-year overall survival (OS) rate of below 20\% after diagnosis, hepatocellular carcinoma (HCC) is one of the most aggressive and least understood cancers worldwide (Wang et al. 2016). The lethality of HCC is mainly due to delayed diagnosis, postoperative recurrence, and limited therapeutic options for advanced-stage HCC. It highlights an urgent need to clarify the potential molecular mechanisms underlying HCC development and develop promising clinical biomarkers (Farazi \& DePinho 2006). Although increasing candidate biomarkers have been proposed to improve HCC diagnosis and prognosis (Singh et al. 2018), few enter into clinical application partly owing to the multiplex attributes and multifaceted roles of the biomarkers.

KPNA2, also known as importin $\alpha-1$, is a member of the karyopherin $\alpha /$ importin $\alpha$ family. It participates in the classical nuclear protein import pathway as an adaptor binding the nuclear localization signal (NLS) of cargo proteins and linking them to importin $\beta$ which ferries the ternary complex through the nuclear pore complex (Goldfarb et al. 2004). Elevated levels of KPNA2 have been observed in a variety of cancers including HCC and were correlated with poor prognosis in most solid tumors (Yoshitake et al. 2011a; Zhou et al. 2017). Our pre-analysis of HCC gene expression data of public databases identified KPNA2 as a unique independent unfavorable predictor for HCC OS and demonstrated its increased expression in early stage of HCC (Supplementary file 1), in accordance with other experimental evidence (Jiang et al. 2014; Yoshitake et al. 2011a). KPNA2 was reported to enhance the proliferation and growth of HCC cells, reduce cellular apoptosis, and promote migration in vitro and in vivo (Guo et al. 2019). No nuclear expression of KPNA2 in non-tumorous liver tissues was observed while nuclear KPNA2 expression was significantly upregulated in HCC tissues, which was associated with a poor prognosis and the risk of recurrence in HCC patients (Jiang et al. 2014). It was reported that KPNA2 might promote HCC cell proliferation by increasing the expression of CCNB2/CDK1 (Gao et al. 2018). Another study suggested that KPNA2 regulated STMN1 by import of E2F1/TFDP1 in liver cancer (Drucker et al. 2019). Additionally, nuclear import of PLAG1 by KPNA2 was essential for the role of KPNA2 in HCC cells (Hu et al. 2014a). HBV pre-S2 mutant large surface protein (LHBS) interacted with KPNA2 and blocked nuclear transport of an essential DNA repair and recombination factor NBS1, inducing genomic instability in HBV-infected hepatocytes and explaining the complex chromosome changes in HCCs (Hsieh et al. 2015). However, there still remains a rather unexplored field underlying KPNA2 molecular evidences and mechanisms in HCC.

In the present study, we performed a comprehensive and multilevel integrative analysis of KPNA2 to explore more signature modalities of KPNA2 in HCC including the genetic and epigenetic regulation of its expression, the landscape of its transcript/protein variants, and its association with immune infiltrations and fatty acid metabolism pathway in HCC. In addition, we constructed two risk models for HCC OS and disease-free survival (DFS) based on the multi-omic data of KPNA2 and evaluated their effectiveness. The laboratory work of testing plasma KPNA2 levels supported its nuclear translocation characteristic. The knowledge deepened the understanding of the deregulation and functioning of such an important biomarker in HCC and provided novel insights into HCC pathogenesis and treatment.

\section{Materials and methods}

\section{Available data and data processing}


80 The clinical features of The Cancer Genome Atlas (TCGA) HCC patients (TCGA-LIHC dataset, 81 named TCGA-HCC in this study, $\mathrm{n}=377$ ) and their transcriptome profiling gene expression RNA82 seq data (371 primary tumors, 2 recurrent tumors, and 50 paired normal liver specimens from 371 83 primary HCC patients) were downloaded from the genomic data common (GDC) portal 84 (https://portal.gdc.cancer.gov/). The clinical features of the 377 patients were shown in Table 1. 85 The expressional counts data TMM (trimmed mean of M-values) were normalized for further analyses and the relative expression data of KPNA2 were extracted. The copy number segments after removing germline copy number variation (370 primary tumors from the 377 patients) and the methylation illuminaMethy 450 data (377 primary tumors and 50 paired normal liver specimens from the 377 patients) from TCGA-HCC dataset were downloaded from Xena UCSC browser (https://xenabrowser.net/), from which KPNA2' data were further extracted. The splice variants of KPNA2 were searched through the Ensembl genome database (http://mar2017.archive.ensembl.org/). The normalized RNA-seq data of the KPNA2 transcript variants in TCGA-HCC dataset were extracted from the TCGA Pan-Cancer gene expression data which was also downloaded from UCSC Xena browser. The mRNA profiles of the KPNA2 transcripts were analyzed between the paired normal and tumor samples of $50 \mathrm{HCC}$ patients. To obtain the information of KPNA2 protein variants in HCC, Human Protein Atlas (HPA), a comprehensive antibody-based protein atlas that displays expression and localization patterns of proteins in a large portion of human tissues and organs (Uhlen et al. 2005), was searched.

\section{Insights into the copy number variation, mutation, and methylation status of KPNA2 gene} in $\mathrm{HCC}$

The correlations of KPNA2 expression with the gene copy number variation (CNV) and methylation status in HCC were investigated by spearman correlation analyses. KPNA2 methylation values between HCC tumors and normal liver tissues were compared by Wilcoxon test. The age-, gender-, and tumor stage- corrected prognostic effects of KPNA2 CNV and KPNA2 methylation level were investigated through ezcox package (Wang et al. 2019) in R3.6.1 software. KPNA2 mutations in TCGA-HCC samples were investigated through the GDC portal (https://portal.gdc.cancer.gov/). Besides, the genes whose mutations might result in KPNA2 expression alteration were identified by the cancer biomarker/target discovery tool muTarget (https://www.mutarget.com/).

110 The variables (CNV and methylation) with significant prognostic effects as well as age, gender, tumor stage, and KPNA2 expression entered into multivariable cox regression analyses to construct the risk models for HCC OS and DFS. The risk model was set as follows:

113 risk score $=\sum_{i=1}^{n} ß(i) * \exp (i)$

114 where $\mathrm{n}$ is the number of selected variables, $\beta(i)$ is the coefficient of the variable $\mathrm{i}$ in the 115 multivariable Cox regression analysis, and $\exp (\mathrm{i})$ is the gene expression, tumor stage, and KPNA2 $116 \mathrm{CNV}$ or methylation value of the $\mathrm{CpG}$ sites. According to the risk scores of the patients, high- and 117 low- risk groups were distinguished according to the median risk score as the threshold. The 118 survival differences between high- and low- risk patients were visualized via Kaplan-Meier 119 survival analysis with log rank test. In the survival analysis, only the patients with OS time or DFS 120 time $>$ one month (30 days) were included. The effectiveness of the prognostic models was also 121 evaluated in male patients and female patients, younger patients (age $<=60 \mathrm{y}$ ) and older (age $>$ 
122 60y) patients, early-stage (stage I and stage II) patients and late-stage (stage III and stage IV)

123 patients respectively. For all the analyses, $p<0.05$ was considered statistically significant.

\section{Exploration of KPNA2 potential functions in HCC}

125 To uncover the potential roles of KPNA2 in HCC, based on the tissue-specific PPI data from the 126 DifferentialNet database (Basha et al. 2018), its liver-specific protein-protein interaction (PPI) 127 network and Kyoto Encyclopedia of Genes and Genomes (KEGG) pathway enrichment of the 128 interacting partners were investigated through NetworkAnalyst (https://www.networkanalyst.ca/) 129 (Zhou et al. 2019). The DifferentialNet database provides a differential view of the human 130 interactome by integrating current data of experimentally-detected PPIs with data of gene 131 expression across tissues. The differential tissue-specific score of an interaction was computed as 132 the difference between its tissue-specific score and its median score across all tissues. For the 133 network construction, only the PPIs that score in the top 15 percentiles and bottom 15 percentiles 134 were selected. To further explore KPNA2's relationship with HCC immunoregulation, the 135 microenvironmental infiltration of eight kinds of immune cells including $\mathrm{T}$ cells $\left(\mathrm{CD}^{+} \mathrm{T}\right.$ cells), $136 \mathrm{CD}^{+} \mathrm{T}$ cells, cytotoxic lymphocytes, NK cells, B lymphocytes, the cells originating from 137 monocytes (monocytic lineage), myeloid dendritic cells, and neutrophils, plus two kinds of stroma 138 cells (endothelial cells and fibroblasts) in TCGA-HCC patients were evaluated with 139 Microenvironment Cell Populations-counter (MCP-counter) method (Becht et al. 2016). Their 140 correlations with KPNA2 expression and HCC prognosis were investigated. In addition, KPNA2 141 expression in the immune infiltration cells including $\mathrm{B}$ cells, CD4 ${ }^{+} \mathrm{T}$ cells, $\mathrm{CD} 8^{+} \mathrm{T}$ cells, 142 macrophages, and NK cells were compared between HCC and normal liver tissues in TCGA-HCC 143 dataset via GEPIA (http://gepia2021.cancer-pku.cn/) with the one-way analysis of variance 144 (ANOVA) function.

\section{Investigation of KPNA2 association with fatty acid metabolism in HCC}

146 As the liver plays crucial roles in fatty acid metabolism and dysregulations of fatty acids are related 147 to fatty liver diseases and liver cancer (Barbier-Torres et al. 2020; Seo et al. 2020), we speculated 148 that there might be connection between KPNA2 expression and fatty acid metabolism in liver. 149 Herein, the effect of low fat diet on KPNA2 expression was evaluated in liver samples from eight 150 individuals including four interference groups (with low-fat diet) and four control groups (with their habitual diet) in the GEO dataset GSE7117 using GEO2R (https://www.ncbi.nlm.nih.gov/geo/info/geo2r.html), an interactive web tool that allows users to compare two or more groups of samples in a GEO series. Furthermore, in consideration of the relationship of fatty acid dysregulation with HCC (Che et al. 2019; Montagner et al. 2019), the correlations of KPNA2 with the genes of fatty acid metabolism pathway in TCGA-HCC primary tumors in KEGG database were investigated through Spearman correlation analysis with Hmisc package (https://CRAN.R-project.org/package=Hmisc) in $\mathrm{R}$ software. The potential implication of KPNA2 in fatty acid biosynthesis was evaluated from the purited-corrected Spearman correlations of KPNA2 with the 11 genes whose products were annotated to fatty acid synthase activity (http://amigo.geneontology.org/amigo/term/GO:0004312) via Tumor IMmune Estimation Resource (TIMER) (https://cistrome.shinyapps.io/timer/) and $p<0.05$ was considered significant.

The correlations of KPNA2 with other genes in HCC were also investigated in the TCGAHCC database. Gene set enrichment analysis (GSEA) was performed with the top positively and negatively KPNA2-correlated genes (Spearman's correlation coefficient $>0.3$ or $<-0.3$ ) through a web-based gene set analysis toolkit (http://www.webgestalt.org/\#) to further uncover KPNA2associated pathways and to see if fatty acid metabolism was enriched in the pathways. 
167 Characterization of the variants of KPNA2 transcripts and proteins, and plasma testing

168 The differential expression of KPNA2 transcript variants between paired HCC and normal controls 169 from the 50 HCC patients in the TCGA-HCC dataset was analyzed through paired sample

170

171

172

173

174

175

176

177

178

179

180

181

182

183

184

185

186

187

188

189

190

191

192

193

194

195

196

197

198

199

200

201

202

203

204

205

206

207

208

209

210

211 Wilcoxon rank test and $p<0.05$ was considered statistically significant. To further uncover the potential roles of KPNA2 transcripts in HCC development, the differentially expressed transcripts were evaluated to investigate their correlations with alpha fetoprotein (AFP) and albumin (ALB) gene expression of HCC samples by Spearman correlation analysis. Moreover, multivariable Cox regression analysis of the differentially expressed transcripts, gender, age, and tumor stage as the covariates was performed to investigate their independent prognostic values.

For KPNA2 protein variants, the immunohistochemical staining patterns with the antibodies against different immunogenic fractions of KPNA2 in HCC and normal liver tissues were extracted from the HPA database and analyzed. The protein level of KPNA2 (full-length) in plasma was examined by enzyme linked immunosorbent assay (ELISA). The work involving the plasma specimens was reviewed and approved by the Ethics Committee of Xiangya Hospital of Central South University (approval number: 201801002). All samples were collected with informed consent in accordance with the Declaration of Helsinki. A total of 92 plasma samples with clinical information (51 samples from primary HCC patients and 41 samples from healthy individuals) were enrolled in the study (Supplementary file 2 Table S1). The plasma samples were obtained between July 2018 and December 2018 from Xiangya Hospital of Central South University (Changsha, China) at the time of diagnosis before any therapy. KPNA2 concentration was measured in duplicate using commercially available ELISA kits (abx250558, Abbexa Ltd, UK) according to the manufacturer's instructions. Absorbance was read at $450 \mathrm{~nm}$ with wavelength correction set at $540 \mathrm{~nm}$ using an ELISA plate reader (BioRad, CA, USA). Wilcoxon test was used for two-group comparison. ROC analysis was applied to investigate the diagnostic power of the factor with $\mathrm{pROC}$ package (Robin et al. 2011) in $\mathrm{R}$ software. For the analyses, $p<0.05$ was considered to be statistically significant.

\section{Results}

\section{Genetic and epigenetic regulations of KPNA2 and their prognostic value in HCC}

By correlation analysis, a significant positive correlation of KPNA2 CNV with KPNA2 expression was shown in Figure 1A $(R=0.43, p<0.001)$. Four $\mathrm{CpG}$ sites locating in KPNA2 promoter including cg23206777, cg22429852, cg21018429, and cg21820889 presented negative correlations ( $p<0.05$, Figure 1B-E) with KPNA2 expression respectively in HCC, indicating their potential roles in regulating KPNA2 expression. All of the $13 \mathrm{CpG}$ methylation sites in KPNA2 with location information, KPNA2 expression-correlation values, and $p$ values were showed in Supplementary file 2 Table S2. By comparison analysis of the four sites methylation between HCC tumors and the control groups, lower methylation of cg22429852 and higher methylation of cg23206777 was found in HCC than in the normal, while the other two sites methylation showed no significant difference (Figure 1F-I). It is noteworthy that the negative relationship of the four sites methylation with KPNA2 expression was conducted in the HCC data without normal liver data. Thus we deduced that certain methylation sites might be affected by negative feedback regulation under physiological and pathological processes, and cg22429852 might be the most critical methylation site for KPNA2 upregulation. For KPNA2 gene mutation inquiry in the GDC portal (https://portal.gdc.cancer.gov/), only four patients in TCGA-HCC dataset were found to have KPNA2 mutations and no significant KPNA2 expression difference was revealed between the HCC samples with and without KPNA2 mutation ( $p=0.69$, Supplementary file 2 Figure S1).

Peer) reviewing PDF | (2021:05:61542:2:0:NEW 5 Aug 2021) 
212 As for other gene mutation effects on KPNA2 expression, by muTarget analysis with mutation

213 prevalence set at 3\%, five mutated genes appeared to affect KPNA2 expression. As shown in

214 Supplemental file 2 Figure S2, the mutations of TP53 $(p<0.01)$, DNAH10 $(p<0.01)$, TSC2 $(p<$

$2150.01)$, and RB1 $(p<0.01)$ were associated with KPNA2 upregulation while KPNA2 was more

216 reduced in HCC samples with BAP1 mutations $(p<0.01)$ than in those without BAP1 mutations.

217 When adjusted with age, gender, and tumor stage, the methylation status of two CpG sites

218 of $\operatorname{cg} 23206777(p=0.026)$ and $\operatorname{cg} 17985418(p=0.00258)$ was shown to have a favorable or an

219 unfavorable prognostic effect on HCC OS respectively, while KPNA2 CNV and other KPNA2

$220 \mathrm{CpG}$ sites presented no significant correlation with HCC OS (Supplementary file 2 Table S3). For

221 evaluation of the CNV and $\mathrm{CpG}$ associations with HCC DFS (Supplementary file 2 Table S4),

222 KPNA2 CNV $(p=0.002)$ and the methylation value of $\operatorname{cg} 14898140(p=0.006)$ were shown to

223 have unfavorable prognostic effects on HCC DFS independent of age, gender, and tumor stage

224

225

226

227

228

229

230

231

232

233

234

235

236

237

238

239

240

241

242

243

244

245

246

247

248

249

250

251

252

253

254

255

256 while no significant correlation of other $\mathrm{CpG}$ sites was shown.

Additionally, the risk models for HCC OS and DFS were constructed by multivariable Cox regression analysis individually. As shown in Figure 2A, tumor stage, KPNA2 expression, and cg17985418 methylation were shown to be independent prognostic factors for HCC OS and the risk model was as follows: $R$ isk score $(O S)=0.40271 *$ tumor stage $+5.49197 *$ cg $17985418+0.55453 *$ KPNA2expssion. There was a shorter OS for the HCC patients with high-risk scores than the ones with low-risk scores (Figure 2B). By ROC analysis, an AUC of 0.814 for 1-year survival, 0.788 for 3-year, and 0.733 for 5-year survival was shown respectively in Figure $2 \mathrm{C}$, indicating the effectiveness of the risk model for predicting HCC patients OS. To compare the predictive efficiency for HCC OS between the constitutive risk model and KPNA2 alone, the predictive efficiency of KPNA2 for HCC OS was calculated. By comparison, although there was no significant difference in their AUCs of 1 -year OS (0.814 vs.0.779, $\mathrm{p}=0.113)$, the AUCs of 3 -year $(0.788$ vs. $0.707, p=0.003)$ and 5 -year OS $(0.733$ vs. $0.665, p=0.034)$ of the constitutive model were significantly higher than those of KPNA2 alone (Supplementary file 2 Figure S3). It indicated the superiority of a multivariable risk score model in the prediction of HCC prognosis, especially for the long survival status of the patients.

With regards to HCC DFS (Figure 3), tumor stage, KPNA2 CNV, and cg14898140 methylation were presented to be independent prognostic factors for HCC patients, while the prognostic value of KPNA2 expression was not significant (Figure 3A). The risk model was established as: $\quad$ Risk score $(D F S)=0.489 *$ tumor stage $+0.644 * K P N A 2 \_C N V+11.3 *$ cg14898140). The HCC patients with high-risk scores had a shorter disease-free time than the patients with low-risk scores (Figure 3B). From the ROC analysis, the AUCs for 1-year, 3-year, and 5-year HCC DFS was $0.721,0.670$, and 0.666 respectively (Figure 3C).

Furthermore, the unfavorable prognostic effects of OS high-risk scores were shown in male HCC patients $(p<0.0001$, Figure 4A), female HCC patients $(p<0.0001$, Figure 4B), young HCC patients $(p<0.0001$, Figure 4C), elderly HCC patients ( $p<0.0001$, Figure 4D), early-stage HCC patients $(p<0.0001$, Figure 4E) and late-stage HCC patients $(p=0.00026$, Figure $4 \mathrm{~F})$ individually, indicating that the OS risk score was an independent prognostic factor. Likewise, the HCC patients with DFS high-risk scores had shorter disease-free time than those with the low-risk scores in different gender groups $(p<0.01$, Figure 4G-H), different age groups $(p<0.0001$, Figure 4I-J), and the early-stage group ( $p<0.0001$, Figure $4 \mathrm{~K}$ ). In the late-stage group (Figure 4L), though only one of the 77 late-stage patients was grouped into the low-risk group, it still showed a longer

Peer] reviewing PDF | (2021:05:61542:2:0:NEW 5 Aug 2021) 
257 disease-free time than most of the patients with high-risk scores. It indicated that the prognostic

258 effect of the risk scores for HCC DFS was independent of gender, age, and tumor stage.

\section{Immunoregulatory roles of KPNA2 in HCC}

260 Via NetworkAnalyst, the liver-specific PPI network of KPNA2 was investigated and 68 proteins

261

262

263

264

265

266

267

268

269

270

271

272

273

274

275

276

277

278

279

280

281

282

283

284

285

286

287

288

289

290

291

292

293

294

295

296

297

298

299

300

301 were found to have interactions with KPNA2 (Figure 5A). By KEGG pathway analysis, the interacting proteins were enriched into five significant terms including viral carcinogenesis, focal adhesion, leukocyte transendothelial migration, PI3K-Akt signaling pathway, and adherens junction (Figure 5B), suggesting the association of KPNA2 with HCC immunology.

Further study revealed that KPNA2 expression was positively correlated with infiltration of five kinds of immune cells in HCC including monocytic lineage cells $(R=0.35, p=4 \mathrm{e}-12$, Figure 5C), T cells $(R=0.23, p=1 \mathrm{e}-05$, Figure 5D), B lineage cells $(R=0.14, p=0.0071$, Figure $5 \mathrm{E}), \mathrm{CD}^{+} \mathrm{T}$ cells $(R=0.14, p=0.006$, Figure $5 \mathrm{~F})$, and myeloid dendritic cells $(R=0.13, p=$ 0.014 , Figure $5 \mathrm{G})$. While no significant correlation of KPNA2 expression with cytotoxic lymphocytes $(p>0.05$, Figure 5H), neutrophils $(p>0.05$, Figure 5I), and NK cells $(p>0.05$, Figure 5J) was indicated. As to the two kinds of stroma cells, a negative relationship between endothelial cell infiltration and KPNA2 expression was presented $(R=-0.19, p=0.00033$, Figure $5 \mathrm{~K}$ ), while no significant correlation was shown between KPNA2 expression and the level of fibroblasts $(p>0.05$, Figure 5L). The correlation of KPNA2 with immune cell infiltrations in early (TNM stage I) and middle-late stage (TNM stage II/III/IV) were evaluated separately (Supplementary file 2 Figure S4). Interestingly, the Spearman correlations of the five types of immune infiltration cells with KPNA2 appear statistically significant or more significant in HCC middle-late stage than in the early stage. It indicates that KPNA2' connection with HCC immune infiltration is becoming increasingly apparent during HCC progress. By Cox regression analysis, among the KPNA2-correlated immune cells and stroma cells, monocytic lineage cells and myeloid dendritic cells were associated with HCC OS ( $p<0.05$, Figure 6A), while none presented prognostic significance for HCC DFS ( $p>0.05$, Figure 6B).

GEPIA2021 analysis resulted that among the five kinds of immune cells including B cells, $\mathrm{CD}^{+} \mathrm{T}$ cells, $\mathrm{CD} 8^{+} \mathrm{T}$ cells, macrophages, and NK cells, KPNA2 was lower expressed in $\mathrm{CD}^{+} \mathrm{T}$ cells $(p=0.02)$ and macrophages $(p<0.01)$ in HCC tumors in comparison with that in normal liver tissues, as shown in Figure 7. Considering its important functions in normal cells (Goldfarb et al. 2004), KPNA2 dysregulation might connect to the dysfunction of the immune cells in HCC.

\section{Associations between KPNA2 expression and fatty acid metabolism}

As shown in Figure 8A, following a low-fat hypocaloric diet for 8 weeks was a significant decrease of KPNA2 expression (probes: 201088_at and 211762_s_at from GPL570) in the four obese liver tissues $(p<0.05)$, indicating a negative effect of low-fat diet on KPNA2 expression in liver.

For the association of KPNA2 expression with fat acid metabolism related genes in HCC, among the 42 genes in KEGG fatty acid metabolism pathway, 88.1\% of them (37/42) were shown to be significantly and negatively correlated with KPNA2 expression (Supplementary file 2 Table S5). The top ten negatively correlated genes were shown in Figure 8B. When the KPNA2negatively-correlated genes in fatty acid metabolism pathway were applied to functional enrichment analyses, multiple fatty acids metabolic processes were revealed including fatty acid degradation and beta-oxidation on the top (Figure 8C). As the down-regulation of fatty acid degradation and beta-oxidation might lead to the increase of fatty acid in the liver, a positive correlation between KPNA2 expression and fatty acid level in HCC was deducible. In fact, as shown in Table 2 , nine of the eleven genes $(81.8 \%)$ related to fatty acid synthase activity were

Peer) reviewing PDF | (2021:05:61542:2:0:NEW 5 Aug 2021) 
302 shown to be significantly positively correlated with KPNA2 expression in HCC. All of these

303 results were consistent with the association between low fat diet and KPNA2 decrease in the liver

304 from the beginning analysis.

305

In addition, the correlations of KPNA2 with other genes in HCC were investigated in the

306 TCGA-HCC database. Its top positive and negative correlated genes with Spearman's correlation

307 coefficient above 0.3 or below -0.3 were extracted (Supplementary file 3). In KEGG GSEA result,

308 KPNA2-correlated genes were most positively enriched in cell cycle pathway while negatively 309 associated with a variety of metabolic pathways including fatty acid degradation (Supplementary

310 file 2 Figure S5), consistent with KPNA2 negative involvement in fatty acid degradation in HCC.

311 The landscape of KPNA2 transcripts and protein variants in HCC

312 KPNA2 is encoded by the KPNA2 gene on the chromosome 17 which contains 11 exons spanning

313 approximately $11 \mathrm{~kb}$ in the human genome. The full-length KPNA2 protein (UniProt_P52292)

314 consists of 529 amino acids and weighs around $58 \mathrm{kDa}$, comprising an N-terminal hydrophilic

315 importin $\beta$-binding domain (IBB, from 2 to 60 amino acid), a central hydrophobic region which

316 binds the cargo's NLS (from 142 to 403 amino acid), and a short acidic C-terminus with no

317 reported function. KPNA2 has seven splice transcript variants as shown in Supplementary file 2

318 Figure S6A. Both of ENST00000330459 (amount percentage range: 25.87\%-100\%) and

319 ENST00000537025 (amount percentage range: 0-1.16\%) code for the full length of KPNA2

320 protein, the former of which is the predominant transcript among all the variants (Supplementary

321 file 2 Figure S6B). ENST00000579754 and ENST00000584026 are derived from alternative

322 splicing discarding the rear half of exon 5 and the posterior exons, resulting in two short protein

323 fragments of N-terminal 143 and 134 amino acids respectively, which retain only the IBB domains.

324 ENST00000583392 and ENST00000582898 are two non-coding RNAs (ncRNAs).

325

326

Paired samples Wilcoxon tests revealed that the two long coding transcripts (ENST00000330459 and ENST00000537025) $(p<0.05$, Figure 9A-B) and the two ncRNAs

327

328

329

330

331

332

333

334

335

336

337

338

339

340 (ENST00000583392 and ENST00000582898) $(p<0.05$, Figure 9F-G) were higher expressed in HCC than in the normal controls. No significant expression difference of the two short proteincoding mRNA (ENST00000579754 and ENST00000584026) $(p>0.05$, Figure 9C-D) and the nonsense mediated decay ENST00000583269 ( $p>0.05$, Figure 9E) was shown between the paired normal liver and HCC samples. Among the four differentially expressed transcripts (Supplementary file 2 Table S6), ENST00000330459 $(r=0.223, p=1.583 \mathrm{E}-05)$ and ENST00000582898 ( $r=0.189, p=2.538 \mathrm{E}-04)$ were shown to be positively correlated with AFP expression. In contrast, the negative correlations of ENST00000330459 $(r=-0.280, p=4.384 \mathrm{E}-$ $08)$ and ENST00000583392 $(r=-0.147, p=0.005)$ with ALB expression were shown. Previous studies supported that serum AFP level was positively associated with HCC nuclear KPNA2 expression (Jiang et al. 2014; Yoshitake et al. 2011b). It substantiated KPNA2 involvement in liver dysfunction and HCC development.

When the four differentially expressed transcripts were applied to multivariable Cox regression analysis with gender, age, and tumor stage as variates, tumor stage and the full-length

341 KPNA2 coding transcript ENST00000330459 independently showed unfavorable prognostic

342 significance for HCC OS (Figure 10A) and DFS (Figure 10B). While the other full-length KPNA2

343 coding transcript ENST00000537025, which made up a tiny constitution of the transcript variants,

344 showed a significant favorable prognostic effect on HCC OS (HR $=0.91, p<0.05$, Figure 10A).

345 Although KPNA2 was reported to be upregulated in HCC tissues in a recent proteomic

346 study (Jiang et al. 2019), the distribution of KPNA2 protein variants was unclear. Herein, the

347 immunohistochemical staining patterns of KPNA2 variants between HCC and normal liver

Peer] reviewing PDF | (2021:05:61542:2:0:NEW 5 Aug 2021) 
348

349

350

351

352

353

354

355

356

357

358

359

360

361

362

363

364

365

366

367

368

369

370

371

372

373

374

375

376

377

378

379

380

381

382

383

384

385

386

387

388

389

390

391

392

samples were extracted from HPA database. Two types of KPNA2 antibodies against different immunogenic fragments of KPNA2 resulted in two different staining patterns. Raised against the amino acids 480-529 in KPNA2 C-terminal, CAB015460 antibody reacted solely to the full length KPNA2 (UniProt_P52292), while HPA041270 antibody which was against the immunogenic sequence in N-terminal of KPNA2 detected all the KPNA2 protein variants including the full length KPNA2 and the other two short protein fragments (UniProt_J3KS65 and J3QLL0) which keep N-terminal domain. KPNA2 was stained by CAB015460 moderately in the cytoplasm/membrane with no nuclear staining in all of the three normal liver samples, while showed nuclear staining in all of the six HCC samples which ranged from weak to strong with rare cytoplasmic or membranous staining observed. The representative staining images were showed in Figure 11A and Figure 11B respectively. With the other antibody HPA041270 which indiscriminately detected all the KPNA2 protein variants, the three normal liver samples were extensively stained in both cytoplasm/membrane and nuclei (Figure 11C). The staining differences of KPNA2 between using the two antibodies in the normal liver samples indicated that KPNA2 in the normal nuclei might be the short KPNA2 protein fragments which could only bind with antibody HPA041270, not CAB015460. The short fragment of KPNA2 might play physiological roles in normal liver cells. In the three HCC samples, KPNA2 was more intensive in HCC nuclei (Figure 11D) than in normal nuclei (Figure 11C), indicating that at least a part of KPNA2 variants were translocated to nuclei if not all, and it was most likely the full-length KPNA2 (UniProt P52292).

We tested the full-length KPNA2 protein level in plasma. As shown in Figure 12A, plasma KPNA2 (UniProt_P52292) was shown to be lower in HCC patients than in normal individuals $(p<0.001)$, which might be partly due to the translocation of KPNA2 to nuclei in HCC as described above. By ROC analysis, the AUC for plasma KPNA2 was 0.787 in discriminating HCC from normal controls (Figure 12B). With an optical cutoff value of $7.923 \mathrm{ng} / \mathrm{ml}, 65.9 \%$ (sensitivity) of the $51 \mathrm{HCC}$ patients and $98.0 \%$ (specificity) of the 41 normal controls could be accurately discriminated, indicating the diagnostic power of plasma KPNA2 in HCC.

\section{Discussion}

In our preliminary bioinformatics analysis on public datasets of HCC, KPNA2 was highlighted to be a unique independent predictor for poor OS of HCC among the differentially expressed genes, and the other independent indicator was tumor stage (Supplementary file 1). Although the association with aggressive clinical characteristics of HCC indicated KPNA2 as a potential therapeutic target, targeting of the factor would probably be complicated by its molecular characteristics, dysregulation mechanisms, and multiple cellular processes that are associated with KPNA2.

KPNA2 deregulation mechanisms have been studied at transcriptional and posttranscriptional levels involving transcriptional regulation factors, miRNAs, and long non-coding RNAs (Feng et al. 2016; Xiang et al. 2019; Zan et al. 2019). Our study provided a supplementary viewpoint from genetic and epigenetic perspectives. Chromosomal instability including frequent chromosome gains (1q, 5, 6p, 7, 8q, 17q and 20) and losses (1p, 4q, 6q, 8p, 13q, 16, 17p and 21) was usually described in HCC (Guichard et al. 2012). KPNA2 gene which is located at chromosome $17 \mathrm{q}$ has not been noted for its copy number alteration in HCC. Herein a high frequency of KPNA2 CNV gains was found in HCC and significantly associated with KPNA2 expression, indicating that KPNA2 gene $\mathrm{CNV}$ was a crucial component of its deregulation mechanisms. The four $\mathrm{CpG}$ sites with negative methylation-expression correlation with KPNA2

Peer) reviewing PDF | (2021:05:61542:2:0:NEW 5 Aug 2021) 
393 provided another potential regulatory mechanism for KPNA2 expression. In addition, KPNA2 $394 \mathrm{CNV}$ and methylation status presented as potential biomarkers for HCC OS/DFS prognosis. It is

395

396

397

398

399

400

401

402

403

404

405

406

407

408

409

410

411

412

413

414

415

416

417

418

419

420

421

422

423

424

425

426

427

428

429

430

431

432

433

434

435

436

437

438 noteworthy that neither the $\mathrm{CpG}$ site $\operatorname{cg} 17985418$ nor $\operatorname{cg} 14898140$, a constitute part of the risk model for HCC OS or DFS with an unfavorable prognostic effect respectively, showed any significant relationship with KPNA2 expression. It might be explained that certain CpG sites methylation might represent as DNA methylation patterns and specific features of threedimensional genome architecture which could have an even more important effect on global gene expression than gene silencing by promoter methylation. At this point, the constitutive risk models involving $\mathrm{CpG}$ sites methylation are presumably more predictive for HCC prognosis than KPNA2 expression level alone, which has been calculated and verified in the Result section.

Most studies have focused on identifying the cargo proteins of which KPNA2 mediated nuclear localization. Both oncoproteins and tumor suppressor proteins are nuclear translocated with KPNA2 such as E2F1, OCT4, c-Myc, p53, p27, MDC1, FGF1/2, LEF-1, CHK1, BRCA1, S100A2, S100A6, RECQL, RAC1, p65, JNK1, STAT3, c-Jun, NBS1, and TBP-2 (Han \& Wang 2020; Martinez-Olivera et al. 2018; Zhou et al. 2021). These downstream signaling pathways including PI3K/AKT, P53, MAPK, cell cycle, Wnt/ $\beta$-catenin, and EMT-related pathways affect the growth, invasion, and metastasis of tumors (Han \& Wang 2020). Given that KPNA2 binds to oncoproteins and tumor suppressor proteins indistinctively, it might depend on oncogenic or tumor suppressive signaling and specific space-temporal contexts to distinctively regulate the transport of oncoproteins and tumor suppressor proteins. For instance, KPNA2 was one of candidate target genes of p53 which inhibits KPNA2 in liver cancer to suppress tumor cell growth (Winkler et al. 2014). Moreover, the fragmental KPNA2 variants which lack the NLS-binding domain but retain their ability to bind importin $\beta$ might affect the full-length KPNA2's function through competitively binding to importin $\beta$. KPNA2 could affect carcinogenesis by mistaken translocation of cancer-associated cargo proteins such as c-Myc (Duan et al. 2020) and PLAG1 (Hu et al. 2014a), both of which could be found in the liver-specific KPNA2 PPI network. The nuclear import of PLAG1 by KPNA2 was reported to lead to the enhanced proliferation and invasive activities of HCC cells ( $\mathrm{Hu}$ et al. 2014b). Though KPNA2 has been revealed to bring a variety of malignant characteristics to HCC, its role in HCC immunology was seldom referred to.

Liver is a central immunomodulator that ensures organ and systemic protection while maintaining immunotolerance (Ringelhan et al. 2018). Persistent deregulation of the tightly controlled immunological network leads to necroinflammation, promotion of liver fibrosis, and subsequently the induction of tumorigenesis (Ringelhan et al. 2018). The bioinformatics evidence herein demonstrated the involvement of KPNA2 in HCC immunological network and a positive correlation with five types of HCC-infiltrating immune cells in agreement with the breakdown of liver immunotolerance. By virtue of KPNA2's role in nucleocytoplasmic transport, a possible related mechanism is altered nuclear shuttling of immunological modulators. For instance, NLRP3, a pro-inflammatory molecule involved in inflammasome activation, was recently found to be an interaction partner for KPNA2 (Park et al. 2019), implying the participation of KPNA2 in an inflammatory cascade. On the other hand, KPNA2 expression was up-regulated in response to extracellular immune signals such as TNF $\alpha$, IL-1 $\beta$ and IL-10 (Liu et al. 2015; Tao et al. 2015; Zhang et al. 2019), indicating the interaction of KPNA2 and an inflammatory microenvironment.

Liver is also crucial in fatty acid metabolism (Bhushan et al. 2019; Ordóñez et al. 2019). The negative relationships of KPNA2 with fatty acid degradation and beta-oxidation processes and its positive involvement in fatty acid synthase activity demonstrated KPNA2' role in dysregulation of fatty acid metabolism. Considering low-fat diet effect on KPNA2 decrease in

Peer] reviewing PDF | (2021:05:61542:2:0:NEW 5 Aug 2021) 
439 obese livers, diet control might be beneficial to prevention and treatment of HCC, especially for 440 obese patients.

441

442

Former studies always ignore KPNA2's transcripts and take them as a whole, which might

443

444

445

446

447

448

449

450

451

452

453

454

455

456

457

458

459

460

461

462

463

464

465

466

467

468

469

470

471

472

473

474

475

476

477 skip its genetics background and potential functions. In our analysis, the two full-length KPNA2 transcripts demonstrated independently prognostic significance for HCC OS in a paradoxical way. ENST00000330459 of $1977 \mathrm{bp}$ with a predominant proportion is unfavorable for HCC outcome while ENST00000537025 of 2456bp with a longer 5' leading sequence UTR shows an inverse effect. One of the potential explanations was that the two transcripts coding the same protein might have been experiencing competition during evolution and the longer 5' UTR region has more opportunities to be modified and degraded. Among the KPNA2 protein variants, the canonical fulllength KPNA2 protein (UniProt_P52292) is significantly upregulated in HCC nuclei but is rarely presented in nuclei of non-tumor liver tissues. The abnormal nuclear accumulation of P52292 protein indicates an imbalance in nuclear transport cycle and such changes are associated with carcinogenesis and poor prognosis in a multitude of cancers (Stelma et al. 2016). In contrast, the truncated KPNA2 isoforms which lack the NLS-binding domain but retain their ability to bind importin $\beta$ exist in nuclei of non-tumor liver tissues and in cytoplasm/membrane of $\mathrm{HCC}$ without obvious nuclear translocation, indicating their physiological functions in normal liver cells and failure to transport cargo proteins into nuclei of HCC cells. A previous study evidenced that a truncated KPNA2 lacking the NLS-binding domain predominantly localized in the cytoplasm of breast cancer cells and inhibited nuclear import of p53 (Kim et al. 2000). One possible explanation is that the fragmental KPNA2 variants could affect the full-length KPNA2's function through competitively binding to importin $\beta$. Although detailed functions of the fragmental KPNA2 variants remain unanswered, it is reasonable to speculate that they might be involved in HCC progression by causing inefficient nuclear import of tumor suppressor nuclear proteins.

At last, plasma KPNA2 between HCC patients and healthy groups was compared by ELISA and exhibited lower level with statistical significance in the HCC patients than in the healthy individuals. It was inconsistent with a previous report that serum KPNA2 level was elevated in non-small-cell lung carcinoma patients than in healthy individuals (Wang et al. 2011). Different tumor type is a possible explanation; nuclear translocation of KPNA2 in HCC cells resulting in scarce cytoplasm/membrane expression followed by reduced exocytosis is another reasonable interpretation. Moreover, KPNA2 was lower expressed in the microenvironment of $\mathrm{HCC}$ with venous metastasis than in the metastasis-averse microenvironment of $\mathrm{HCC}$ in a GEO dataset GDS3091 (Budhu et al. 2006), implying that the decreased KPNA2 level surrounding the more aggressive $\mathrm{HCC}$ would result in its lower concentration in the peripheral blood. However, it's worth noting that KPNA2 is expressed in most tissues and organs besides the liver. We examined the plasma KPNA2 in HCC patients and normal individuals given that their other tissues and organs were comparable except the difference in their livers between the two groups. If other tissues and organs weigh a lot in the distinction of KPNA2 release, the plasma KPNA2 might not accurately indicate the change of KPNA2 in HCC. It needs to be further verified in future.

\section{Conclusions}

479 In summary, we explored the molecular characteristics and dysregulation of KPNA2 through in480 depth multi-omics analysis. The CNV and methylation level of KPNA2 gene varied during HCC 481 482 development and predicted the disease outcome. The effectiveness of the risk models for HCC OS and DFS prediction presented the advantage of multi-omics analyses of a specific gene. Furthermore, dissection of KPNA2 transcript variants and protein isoforms broadened KPNA2 
484

485

486

487

488

489

490

491

492

493

494

495

496

497

498

499

500

501

502

503

504

505

506

507

508

509

510

511

512

513

514

515

516

517

518

519

520

521

522

523

524

525

526

527

528

529

530

clinicopathological features. The role of KPNA2 in HCC immunoregulation was highlighted, providing information of immune cell constitutions within HCC microenvironment and for prediction of HCC immune response and constituting a potential resource in anti-tumor immunotherapy. The negative correlations between KPNA2 and most genes in fatty acid metabolism pathway, its positive relationship with fatty acid synthase activity, and low-fat diet effect on KPNA2 decrease in liver indicated the importance of diet control in HCC prevention and treatment. Taken together, the integrative analyses of KPNA2 provided in-depth knowledge of the molecular mechanisms of HCC pathogenesis and explored promising molecular markers and signatures for HCC prognosis and therapy.

\section{ACKNOWLEGMENTS}

We thank Dr. JM Zeng from University of Macau and his team for their help in the bioinformatics analysis.

\section{REFERENCES}

Barbier-Torres L, Fortner KA, Iruzubieta P, Delgado TC, Giddings E, Chen Y, Champagne D, Fernández-Ramos D, Mestre D, Gomez-Santos B, Varela-Rey M, de Juan VG, Fernández-Tussy P, Zubiete-Franco I, García-Monzón C, González-Rodríguez Á, Oza D, Valença-Pereira F, Fang Q, Crespo J, Aspichueta P, Tremblay F, Christensen BC, Anguita J, Martínez-Chantar ML, and Rincón M. 2020. Silencing hepatic MCJ attenuates non-alcoholic fatty liver disease (NAFLD) by increasing mitochondrial fatty acid oxidation. Nat Commun 11:3360. 10.1038/s41467-020-16991-2

Basha O, Shpringer R, Argov CM, and Yeger-Lotem E. 2018. The DifferentialNet database of differential proteinprotein interactions in human tissues. Nucleic Acids Res 46:D522-d526. 10.1093/nar/gkx981

Becht E, Giraldo NA, Lacroix L, Buttard B, Elarouci N, Petitprez F, Selves J, Laurent-Puig P, Sautès-Fridman C, Fridman WH, and de Reyniès A. 2016. Estimating the population abundance of tissue-infiltrating immune and stromal cell populations using gene expression. Genome Biol 17:218. 10.1186/s13059-016-1070-5

Bhushan B, Banerjee S, Paranjpe S, Koral K, Mars WM, Stoops JW, Orr A, Bowen WC, Locker J, and Michalopoulos GK. 2019. Pharmacologic Inhibition of Epidermal Growth Factor Receptor Suppresses Nonalcoholic Fatty Liver Disease in a Murine Fast-Food Diet Model. Hepatology 70:1546-1563. 10.1002/hep.30696

Budhu A, Forgues M, Ye QH, Jia HL, He P, Zanetti KA, Kammula US, Chen Y, Qin LX, Tang ZY, and Wang XW. 2006. Prediction of venous metastases, recurrence, and prognosis in hepatocellular carcinoma based on a unique immune response signature of the liver microenvironment. Cancer Cell 10:99-111. 10.1016/j.ccr.2006.06.016

Che L, Paliogiannis P, Cigliano A, Pilo MG, Chen X, and Calvisi DF. 2019. Pathogenetic, Prognostic, and Therapeutic Role of Fatty Acid Synthase in Human Hepatocellular Carcinoma. Front Oncol 9:1412. 10.3389/fonc.2019.01412

Drucker E, Holzer K, Pusch S, Winkler J, Calvisi DF, Eiteneuer E, Herpel E, Goeppert B, Roessler S, Ori A, Schirmacher P, Breuhahn K, and Singer S. 2019. Karyopherin a2-dependent import of E2F1 and TFDP1 maintains protumorigenic stathmin expression in liver cancer. Cell Commun Signal 17:159. 10.1186/s12964-019-0456$\mathrm{x}$

Duan M, Hu F, Li D, Wu S, and Peng N. 2020. Silencing KPNA2 inhibits IL-6-induced breast cancer exacerbation by blocking NF-KB signaling and c-Myc nuclear translocation in vitro. Life Sci 253:117736. 10.1016/j.Ifs.2020.117736

Farazi PA, and DePinho RA. 2006. Hepatocellular carcinoma pathogenesis: from genes to environment. Nat Rev Cancer 6:674-687. 10.1038/nrc1934

Feng N, Ching T, Wang Y, Liu B, Lin H, Shi O, Zhang X, Zheng M, Zheng X, Gao M, Zheng ZJ, Yu H, Garmire L, and Qian B. 2016. Analysis of Microarray Data on Gene Expression and Methylation to Identify Long Non-coding RNAs in Non-small Cell Lung Cancer. Sci Rep 6:37233. 10.1038/srep37233

Gao CL, Wang GW, Yang GQ, Yang H, and Zhuang L. 2018. Karyopherin subunit- $\alpha 2$ expression accelerates cell cycle

Peer] reviewing PDF | (2021:05:61542:2:0:NEW 5 Aug 2021) 
531

532

533

534

535

536

537

538

539

540

541

542

543

544

545

546

547

548

549

550

551

552

553

554

555

556

557

558

559

560

561

562

563

564

565

566

567

568

569

570

571

572

573

574

575

576

577

578

579

580

581

582

583

progression by upregulating CCNB2 and CDK1 in hepatocellular carcinoma. Oncol Lett 15:2815-2820. 10.3892/ol.2017.7691

Goldfarb DS, Corbett AH, Mason DA, Harreman MT, and Adam SA. 2004. Importin alpha: a multipurpose nucleartransport receptor. Trends Cell Biol 14:505-514. 10.1016/j.tcb.2004.07.016

Guichard C, Amaddeo G, Imbeaud S, Ladeiro Y, Pelletier L, Maad IB, Calderaro J, Bioulac-Sage P, Letexier M, Degos F, Clement B, Balabaud C, Chevet E, Laurent A, Couchy G, Letouze E, Calvo F, and Zucman-Rossi J. 2012. Integrated analysis of somatic mutations and focal copy-number changes identifies key genes and pathways in hepatocellular carcinoma. Nat Genet 44:694-698. 10.1038/ng.2256

Guo X, Wang Z, Zhang J, Xu Q, Hou G, Yang Y, Dong C, Liu G, Liang C, Liu L, Zhou W, and Liu H. 2019. Upregulated KPNA2 promotes hepatocellular carcinoma progression and indicates prognostic significance across human cancer types. Acta Biochim Biophys Sin (Shanghai) 51:285-292. 10.1093/abbs/gmz003

Han Y, and Wang X. 2020. The emerging roles of KPNA2 in cancer. Life Sci 241:117140. 10.1016/j.Ifs.2019.117140

Hsieh YH, Chang YY, Su IJ, Yen CJ, Liu YR, Liu RJ, Hsieh WC, Tsai HW, Wang LH, and Huang W. 2015. Hepatitis B virus pre-S2 mutant large surface protein inhibits DNA double-strand break repair and leads to genome instability in hepatocarcinogenesis. J Pathol 236:337-347. 10.1002/path.4531

Hu ZY, Yuan SX, Yang Y, Zhou WP, and Jiang H. 2014a. Pleomorphic adenoma gene 1 mediates the role of karyopherin alpha 2 and has prognostic significance in hepatocellular carcinoma. J Exp Clin Cancer Res 33:61. 10.1186/s13046-014-0061-1

Hu ZY, Yuan SX, Yang Y, Zhou WP, and Jiang H. 2014b. Pleomorphic adenoma gene 1 mediates the role of karyopherin alpha 2 and has prognostic significance in hepatocellular carcinoma. J Exp Clin Cancer Res 33:61. 10.1186/s13046-014-0061-1

Jiang P, Tang Y, He L, Tang H, Liang M, Mai C, Hu L, and Hong J. 2014. Aberrant expression of nuclear KPNA2 is correlated with early recurrence and poor prognosis in patients with small hepatocellular carcinoma after hepatectomy. Med Oncol 31:131. 10.1007/s12032-014-0131-4

Jiang $Y$, Sun $A$, Zhao $Y$, Ying $W$, Sun $H$, Yang $X$, Xing B, Sun W, Ren L, Hu B, Li C, Zhang L, Qin G, Zhang $M$, Chen $N$, Zhang $M$, Huang $Y$, Zhou J, Zhao Y, Liu M, Zhu X, Qiu Y, Sun Y, Huang C, Yan M, Wang M, Liu W, Tian F, Xu H, Zhou J, Wu Z, Shi T, Zhu W, Qin J, Xie L, Fan J, Qian X, and He F. 2019. Proteomics identifies new therapeutic targets of early-stage hepatocellular carcinoma. Nature 567:257-261. 10.1038/s41586-019-0987-8

Kim IS, Kim DH, Han SM, Chin MU, Nam HJ, Cho HP, Choi SY, Song BJ, Kim ER, Bae YS, and Moon YH. 2000. Truncated form of importin alpha identified in breast cancer cell inhibits nuclear import of p53. J Biol Chem 275:2313923145. 10.1074/jbc.M909256199

Liu Z, Zhang D, Sun C, Tao R, Xu X, Xu L, Cheng H, Xiao M, and Wang Y. 2015. KPNA2 Contributes to the Inflammatory Processes in Synovial Tissue of Patients with Rheumatoid Arthritis and SW982 Cells. Inflammation 38:22242234. 10.1007/s10753-015-0205-2

Martinez-Olivera R, Datsi A, Stallkamp M, Köller M, Kohtz I, Pintea B, and Gousias K. 2018. Silencing of the nucleocytoplasmic shuttling protein karyopherin a2 promotes cell-cycle arrest and apoptosis in glioblastoma multiforme. Oncotarget 9:33471-33481. 10.18632/oncotarget.26033

Montagner A, Le Cam L, and Guillou H. 2019. $\beta$-catenin oncogenic activation rewires fatty acid catabolism to fuel hepatocellular carcinoma. Gut 68:183-185. 10.1136/gutjnl-2018-316557

Ordóñez YF, Abad JL, Aseeri M, Casas J, Garcia V, Casasampere M, Schuchman EH, Levade T, Delgado A, Triola G, and Fabrias G. 2019. Activity-Based Imaging of Acid Ceramidase in Living Cells. J Am Chem Soc 141:7736-7742. 10.1021/jacs.8b11687

Park SH, Ham S, Lee A, Möller A, and Kim TS. 2019. NLRP3 negatively regulates Treg differentiation through Kpna2mediated nuclear translocation. J Biol Chem 294:17951-17961. 10.1074/jbc.RA119.010545

Ringelhan M, Pfister D, O'Connor T, Pikarsky E, and Heikenwalder M. 2018. The immunology of hepatocellular carcinoma. Nat Immunol 19:222-232. 10.1038/s41590-018-0044-z

Robin X, Turck N, Hainard A, Tiberti N, Lisacek F, Sanchez JC, and Müller M. 2011. pROC: an open-source package for $\mathrm{R}$ and $\mathrm{S}+$ to analyze and compare ROC curves. BMC Bioinformatics 12:77. 10.1186/1471-2105-12-77

Seo J, Jeong DW, Park JW, Lee KW, Fukuda J, and Chun YS. 2020. Fatty-acid-induced FABP5/HIF-1 reprograms lipid metabolism and enhances the proliferation of liver cancer cells. Commun Biol 3:638. 10.1038/s42003-02001367-5

Singh AK, Kumar R, and Pandey AK. 2018. Hepatocellular Carcinoma: Causes, Mechanism of Progression and Biomarkers. Curr Chem Genom Transl Med 12:9-26. 10.2174/2213988501812010009

Peer) reviewing PDF | (2021:05:61542:2:0:NEW 5 Aug 2021) 
584

585

586

587

588

589

590

591

592

593

594

595

596

597

598

599

600

601

602

603

604

605

606

607

608

609

610

611

612

613

614

615

616

617

618

619

620

621

622

623

624

625

626

627

628

629

630

631

632

633

Stelma T, Chi A, van der Watt PJ, Verrico A, Lavia P, and Leaner VD. 2016. Targeting nuclear transporters in cancer: Diagnostic, prognostic and therapeutic potential. IUBMB Life 68:268-280. 10.1002/iub.1484

Tao R, Xu X, Sun C, Wang Y, Wang S, Liu Z, Zhai L, Cheng H, Xiao M, and Zhang D. 2015. KPNA2 interacts with P65 to modulate catabolic events in osteoarthritis. Exp Mol Pathol 99:245-252. 10.1016/j.yexmp.2015.07.007

Uhlen M, Bjorling E, Agaton C, Szigyarto CA, Amini B, Andersen E, Andersson AC, Angelidou P, Asplund A, Asplund C, Berglund L, Bergstrom K, Brumer H, Cerjan D, Ekstrom M, Elobeid A, Eriksson C, Fagerberg L, Falk R, Fall J, Forsberg M, Bjorklund MG, Gumbel K, Halimi A, Hallin I, Hamsten C, Hansson M, Hedhammar M, Hercules G, Kampf C, Larsson K, Lindskog M, Lodewyckx W, Lund J, Lundeberg J, Magnusson K, Malm E, Nilsson P, Odling J, Oksvold P, Olsson I, Oster E, Ottosson J, Paavilainen L, Persson A, Rimini R, Rockberg J, Runeson $M$, Sivertsson A, Skollermo A, Steen J, Stenvall M, Sterky F, Stromberg S, Sundberg M, Tegel H, Tourle S, Wahlund E, Walden A, Wan J, Wernerus H, Westberg J, Wester K, Wrethagen U, Xu LL, Hober S, and Ponten F. 2005. A human protein atlas for normal and cancer tissues based on antibody proteomics. $\mathrm{Mol} \mathrm{Cell}$ Proteomics 4:1920-1932. 10.1074/mcp.M500279-MCP200

Wang $\mathrm{Cl}$, Wang CL, Wang CW, Chen CD, Wu CC, Liang Y, Tsai YH, Chang YS, Yu JS, and Yu CJ. 2011. Importin subunit alpha-2 is identified as a potential biomarker for non-small cell lung cancer by integration of the cancer cell secretome and tissue transcriptome. Int J Cancer 128:2364-2372. 10.1002/ijc.25568

Wang S, Sun H, Xie Z, Li J, Hong G, Li D, Mallampati S, Zhou X, Zhou C, Zhang H, Cheng Z, Shan H, and Ma H. 2016. Improved survival of patients with hepatocellular carcinoma and disparities by age, race, and socioeconomic status by decade, 1983-2012. Oncotarget 7:59820-59833. 10.18632/oncotarget.10930

Wang S, Zhang J, He Z, Wu K, and Liu XS. 2019. The predictive power of tumor mutational burden in lung cancer immunotherapy response is influenced by patients' sex. Int J Cancer 145:2840-2849. 10.1002/ijc.32327

Winkler J, Ori A, Holzer K, Sticht C, Dauch D, Eiteneuer EM, Pinna F, Geffers R, Ehemann V, Andres-Pons A, Breuhahn $\mathrm{K}$, Longerich T, Bermejo JL, Gretz N, Zender L, Schirmacher P, Beck M, and Singer S. 2014. Prosurvival function of the cellular apoptosis susceptibility/importin- $\alpha 1$ transport cycle is repressed by p53 in liver cancer. Hepatology 60:884-895. 10.1002/hep.27207

Xiang S, Wang Z, Ye Y, Zhang F, Li H, Yang Y, Miao H, Liang H, Zhang Y, Jiang L, Hu Y, Zheng L, Liu X, and Liu Y. 2019. E2F1 and E2F7 differentially regulate KPNA2 to promote the development of gallbladder cancer. Oncogene 38:1269-1281. 10.1038/s41388-018-0494-7

Yoshitake K, Tanaka S, Mogushi K, Aihara A, Murakata A, Matsumura S, Mitsunori Y, Yasen M, Ban D, Noguchi N, Irie T, Kudo A, Nakamura N, Tanaka H, and Arii S. 2011a. Importin-alpha1 as a novel prognostic target for hepatocellular carcinoma. Ann Surg Oncol 18:2093-2103. 10.1245/s10434-011-1569-7

Yoshitake K, Tanaka S, Mogushi K, Aihara A, Murakata A, Matsumura S, Mitsunori Y, Yasen M, Ban D, Noguchi N, Irie T, Kudo A, Nakamura N, Tanaka H, and Arii S. 2011b. Importin- $\alpha 1$ as a novel prognostic target for hepatocellular carcinoma. Ann Surg Oncol 18:2093-2103. 10.1245/s10434-011-1569-7

Zan Y, Wang B, Liang L, Deng Y, Tian T, Dai Z, and Dong L. 2019. MicroRNA-139 inhibits hepatocellular carcinoma cell growth through down-regulating karyopherin alpha 2. J Exp Clin Cancer Res 38:182. 10.1186/s13046-019$1175-2$

Zhang Z, Huang X, Li J, Fan H, Yang F, Zhang R, Yang Y, Feng S, He D, Sun W, and Xin T. 2019. Interleukin 10 promotes growth and invasion of glioma cells by up-regulating KPNA 2 in vitro. J Cancer Res Ther 15:927-932. 10.4103/jcrt.JCRT_284_19

Zhou G, Soufan O, Ewald J, Hancock REW, Basu N, and Xia J. 2019. NetworkAnalyst 3.0: a visual analytics platform for comprehensive gene expression profiling and meta-analysis. Nucleic Acids Res 47:W234-w241. 10.1093/nar/gkz240

Zhou KX, Huang S, Hu LP, Zhang XL, Qin WT, Zhang YL, Yao LL, Yu Y, Zhou YQ, Zhu L, Ji J, and Zhang ZG. 2021. Increased Nuclear Transporter KPNA2 Contributes to Tumor Immune Evasion by Enhancing PD-L1 Expression in PDAC. J Immunol Res 2021:6694392. 10.1155/2021/6694392

Zhou LN, Tan Y, Li P, Zeng P, Chen MB, Tian Y, and Zhu YQ. 2017. Prognostic value of increased KPNA2 expression in some solid tumors: A systematic review and meta-analysis. Oncotarget 8:303-314. 10.18632/oncotarget.13863

Peer) reviewing PDF | (2021:05:61542:2:0:NEW 5 Aug 2021) 


\section{Table 1 (on next page)}

Clinical features of the $377 \mathrm{HCC}$ patients from TCGA database.

HCC, hepatocellular carcinoma; NA, not available. 
1

\begin{tabular}{|c|c|}
\hline Variables & Case, n (\%) \\
\hline \multicolumn{2}{|l|}{ Age at diagnosis (yr.) } \\
\hline$<60$ & $172(45.6 \%)$ \\
\hline$\geq 60$ & $204(54.1 \%)$ \\
\hline NA & $1(0.3 \%)$ \\
\hline \multicolumn{2}{|l|}{ Gender } \\
\hline Male & $255(67.6 \%)$ \\
\hline Female & $122(32.4 \%)$ \\
\hline \multicolumn{2}{|l|}{ TNM stage } \\
\hline I & $175(46.4 \%)$ \\
\hline II & $87(23.1 \%)$ \\
\hline III & $86(22.8 \%)$ \\
\hline IV & $5(1.3 \%)$ \\
\hline NA & $24(6.4 \%)$ \\
\hline \multicolumn{2}{|l|}{ Pathologic grade } \\
\hline G1 & $55(14.6 \%)$ \\
\hline G2 & $180(47.7 \%)$ \\
\hline G3 & $124(32.9 \%)$ \\
\hline G4 & $13(3.4 \%)$ \\
\hline NA & $5(1.3 \%)$ \\
\hline \multicolumn{2}{|l|}{ Race } \\
\hline White & $187(49.6 \%)$ \\
\hline Asian & $161(42.7 \%)$ \\
\hline Black or African American & $17(4.5 \%)$ \\
\hline American Indian or Alaska Native & $2(0.5 \%)$ \\
\hline NA & $10(2.7 \%)$ \\
\hline \multicolumn{2}{|l|}{ Survival status } \\
\hline Alive & $244(64.7 \%)$ \\
\hline Dead & $132(35.0 \%)$ \\
\hline NA & $1(0.3 \%)$ \\
\hline
\end{tabular}

2 


\section{Table 2 (on next page)}

Correlations between KPNA2 and the genes related to fatty acid synthase activity in HCC.

HCC, hepatocellular carcinoma; ${ }^{*}, p<0.05 ;{ }^{*}, p<0.01$. For the analyses, the tumor puritycorrelated Spearman correlation was evaluated and $p<0.05$ was considered significant. 


\begin{tabular}{lll}
\hline & & \\
\hline Genes related to fatty acid synthase activity & Correlation & P value \\
\hline ELOVL1 & 0.597 & $9.816 \mathrm{E}-35^{* *}$ \\
ELOVL2 & 0.219 & $4.046 \mathrm{E}-05^{* *}$ \\
ELOVL3 & 0.397 & $1.806 \mathrm{E}-14^{* *}$ \\
ELOVL4 & 0.380 & $2.776 \mathrm{E}-13^{* *}$ \\
ELOVL5 & 0.403 & $6.207 \mathrm{E}-15^{* *}$ \\
ELOVL6 & 0.161 & $2.776 \mathrm{E}-03^{* *}$ \\
ELOVL7 & 0.475 & $8.444 \mathrm{E}-21^{* *}$ \\
FASN & 0.338 & $1.160 \mathrm{E}-10^{* *}$ \\
MCAT & 0.068 & 0.209 \\
OLAH & 0.013 & 0.808 \\
OXSM & 0.134 & $0.013^{*}$ \\
\hline
\end{tabular}

2 


\section{Figure 1}

Correlation of KPNA2 CNV and KPNA2 methylation with KPNA2 expression in HCC.

(A) Significant positive correlation between KPNA2 CNV and KPNA2 expression. (B-E) Significant negative correlations between KPNA2 expression and methylation level of cg23206777, cg22429852, cg21018429, and cg21820889, respectively. (F) Hyper methylation of cg23206777 on KPNA2 gene in HCC tumors than in the normal tissues. (G-H) No significant difference of methylation status of cg21018429 and cg21820889 on KPNA2 gene between HCC tumors and normal livers, respectively. (I) Lower methylation of cg22429852 on KPNA2 gene in HCC tumors than in the normal tissues. For (A-E), $x$-axis represented the relative expression of KPNA2 $\left[\log _{2}(T M M+0.001)\right]$ in HCC samples while the $y$ axis indicated CNV or methylation level (beta value) of the $\mathrm{CpG}$ sites. For (F-I), $\mathrm{x}$-axis represented samples of different groups and $y$-axis represented methylation value of KPNA2 gene. Spearman's correlation analysis and Wilcoxon test were used for the analyses and $p<$ 0.05 was considered significant. CNV, copy number variation; TMM, trimmed mean of Mvalues. 

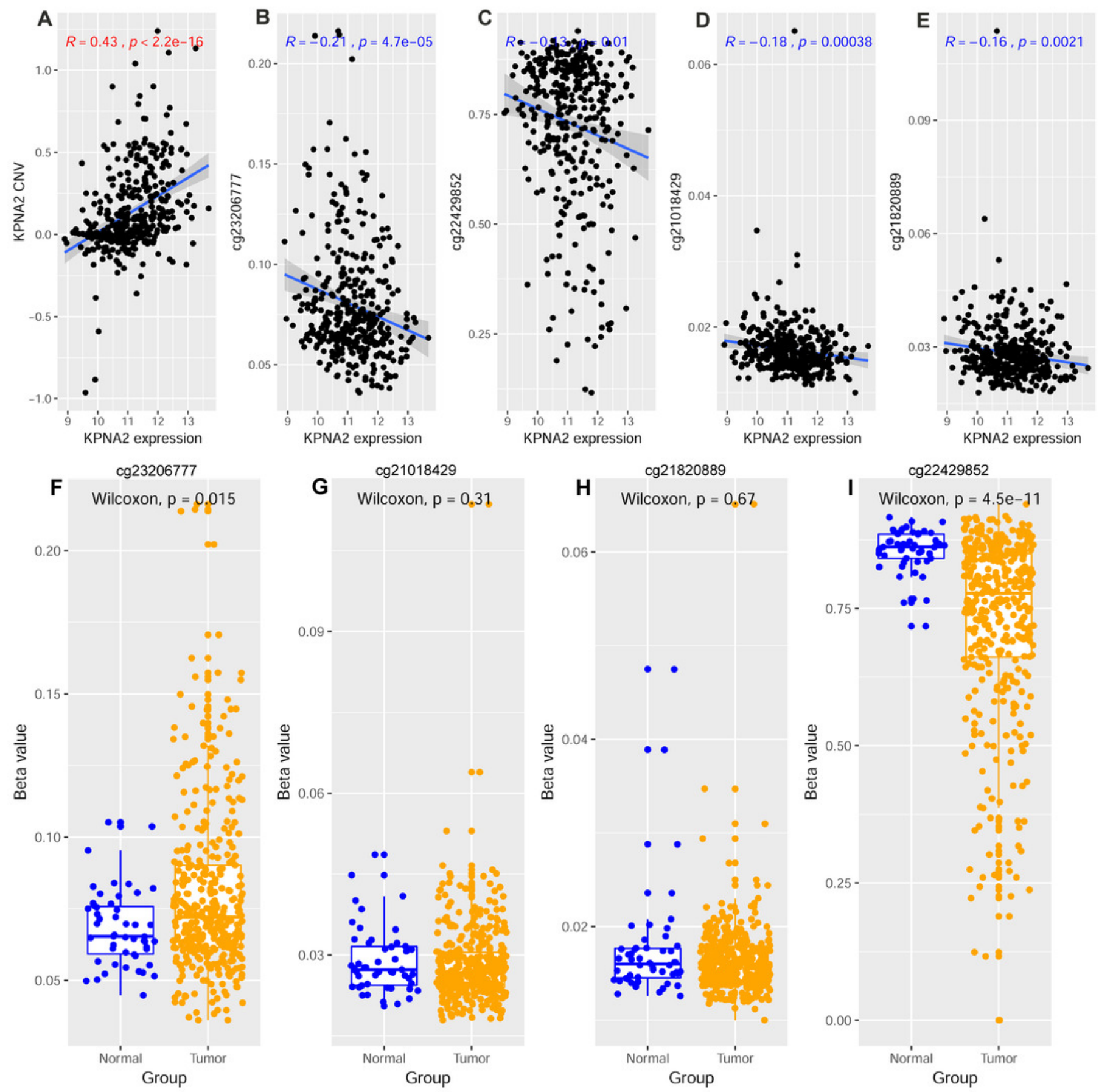


\section{Figure 2}

Construction and evaluation of HCC OS risk model with KPNA2 data and clinical features.

(A) Tumor stage, KPNA2 expression, and cg17985418 methylation were independent prognostic factors for HCC OS and they were selected for the risk model construction. (B) According to the risk model, the HCC patients with high-risk scores presented shorter OS than the ones with low-risk scores. (C) The OS risk model could discriminate the 1-year, 3year, and 5-year survival status of the HCC patients effectively. Multivariable Cox regression analysis was used for the risk model construction. Kaplan-Meier survival analysis with log rank test was used for the survival comparison between high and low risk groups. ROC analysis was used to evaluated the effectiveness of the OS risk model in OS status prediction. OS, overall survival; ROC, Receiver operating characteristic. For all the analyses, $p<0.05$ was considered significant. 


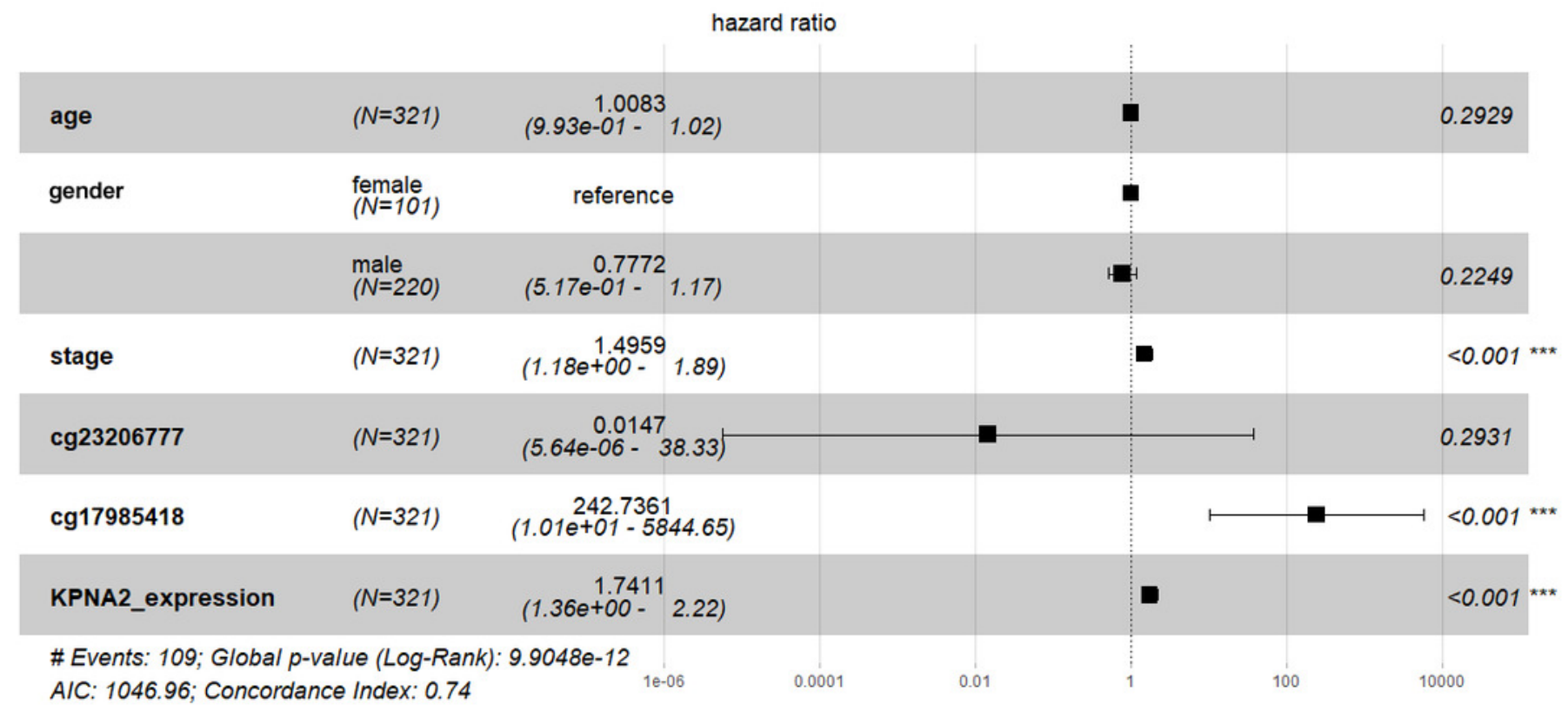

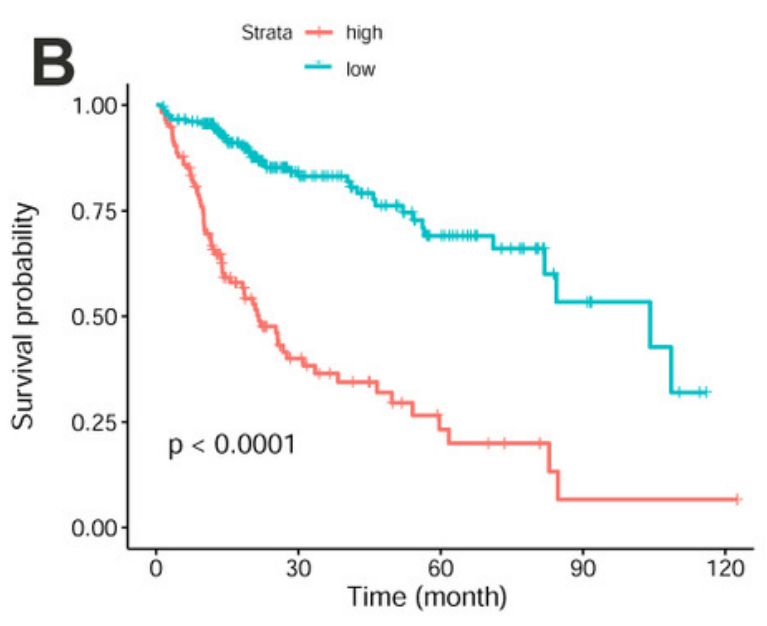

Number at risk

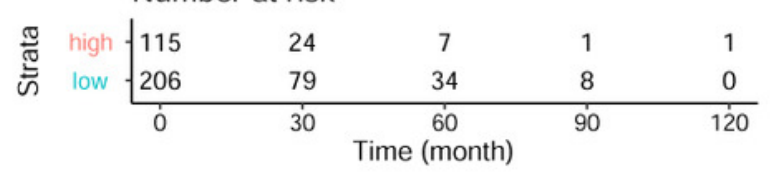

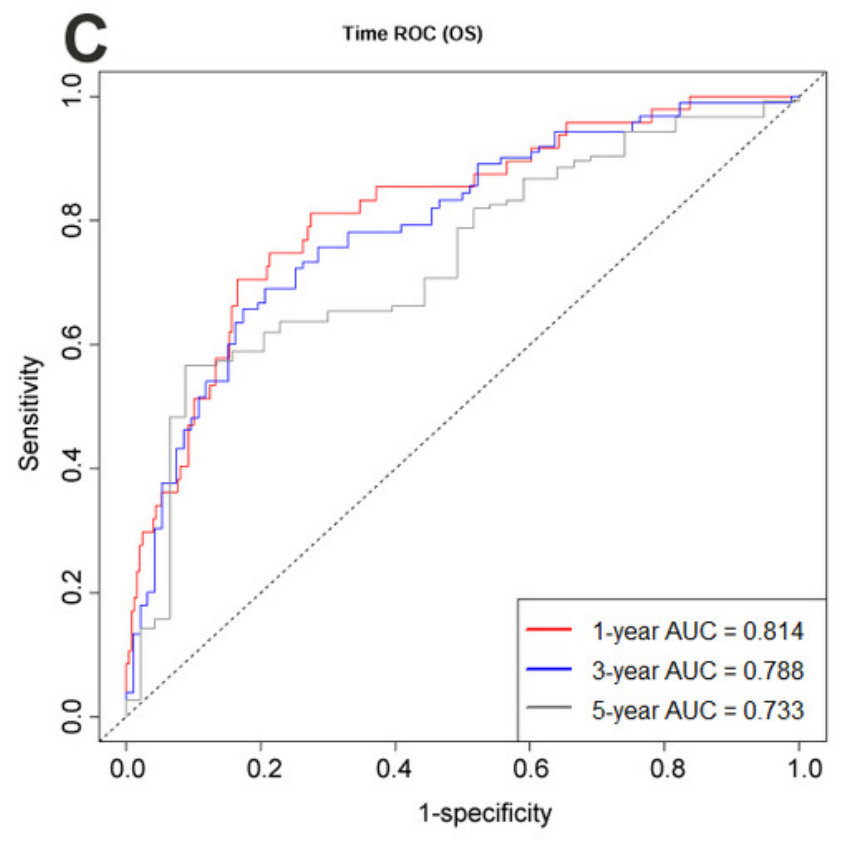




\section{Figure 3}

Construction and evaluation of HCC DFS risk model with KPNA2 data and clinical features.

(A) Tumor stage, KPNA2 CNV, and cg14898140 methylation were independent prognostic factors for HCC DFS and they were selected for the risk model construction. (B) According to the DFS risk model, the HCC patients with high risk scores presented shorter disease-free time than the ones with low risk scores. (C) The DFS risk model could discriminate the 1-year, 3-year, and 5-year DFS status of the HCC patients effectively. Multivariable Cox regression analysis was used for the risk model construction. Kaplan-Meier survival analysis with log rank test was used for the survival comparison between high and low risk groups. ROC analysis was used to evaluated the effectiveness of the DFS risk model in DFS status prediction. DFS, disease-free survival; CNV, copy number variation; ROC, Receiver operating characteristic. For all the analyses, $p<0.05$ was considered significant. 


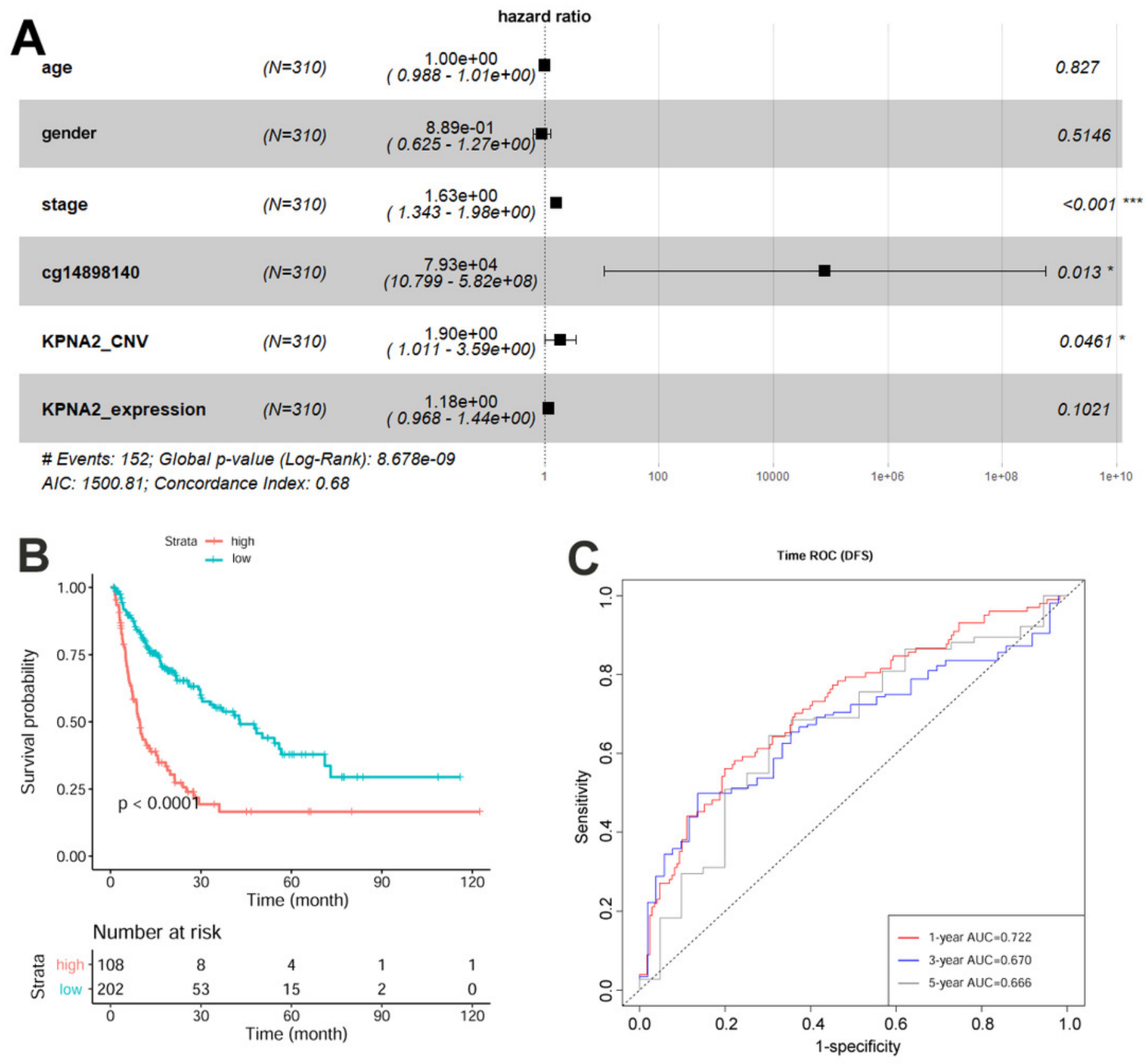




\section{Figure 4}

Independent prognostic effects of the OS and DFS risk models in HCC patients.

Independent prognostic effects of the OS and DFS risk models in HCC patients. (A-B)

Comparing with low-risk scores, high risk scores indicated shorter OS regardless of gender.

(C-D) Comparing with low-risk scores, high risk scores indicated shorter OS regardless of age.

(E-F) Comparing with low-risk scores, high risk scores indicated shorter OS regardless of HCC stage. (G-H) Comparing with low-risk scores, high risk scores indicated shorter disease-free time regardless of gender. (I-J) Comparing with low-risk scores, high risk scores indicated shorter disease-free time regardless of age. (K-L) Comparing with low-risk scores, high risk scores indicated shorter disease-free regardless of HCC stage. OS, overall survival; DFS, disease-free survival. Kaplan-Meier survival analysis with log rank test was used for survival analysis and $p<0.05$ was considered significant. 

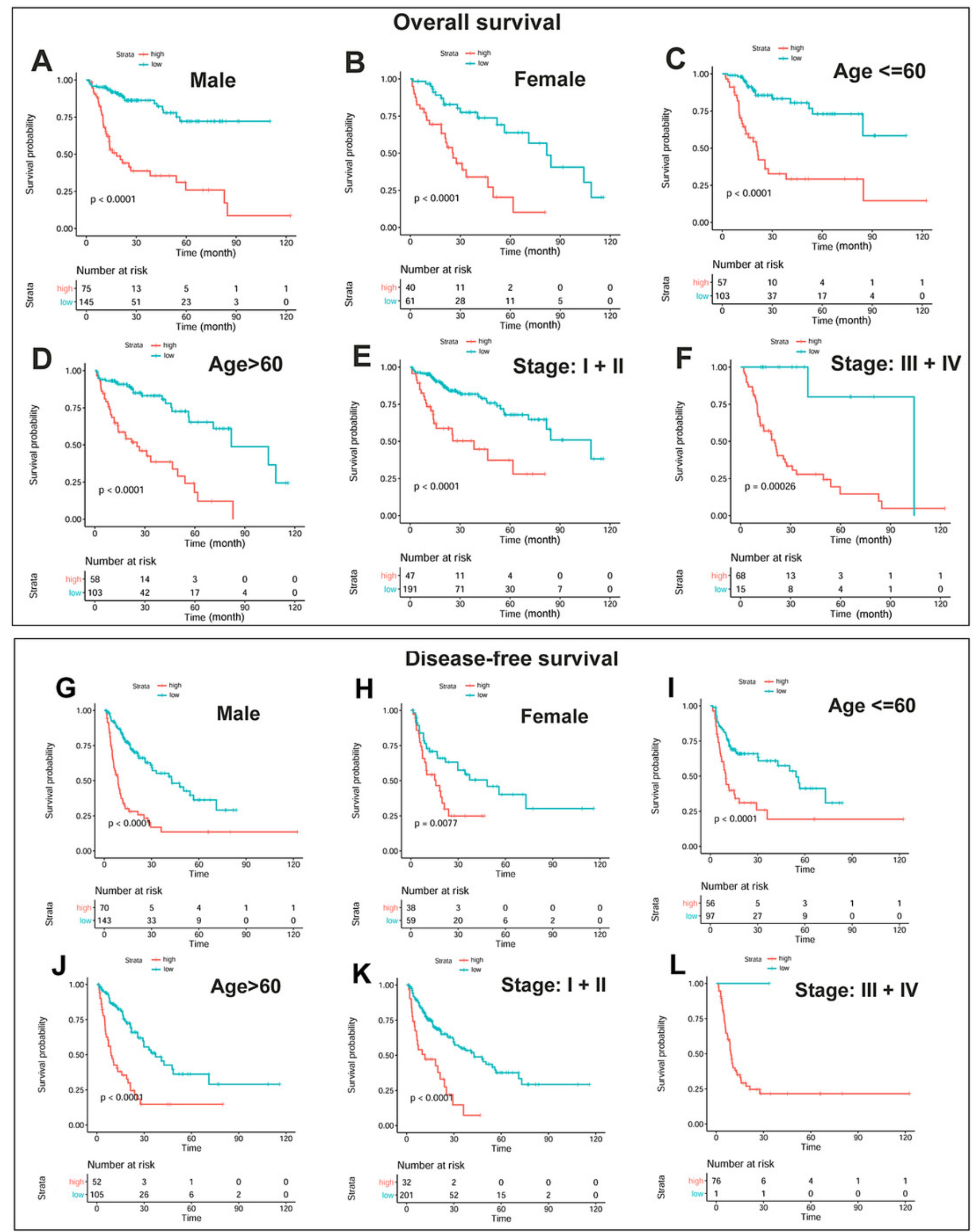


\section{Figure 5}

Liver specific PPI network of KPNA2 and the correlation of KPNA2 with immune response.

(A) The PPI network of KPNA2 in liver. The blue dots genes participated in the pathways in

(B). (B) The KEGG pathways that the genes in KPNA2 PPI network were enriched. (C-G) The significant positive correlations of KPNA2 expression with infiltration of monocytic lineage cells, T cells, B lineage cells, CD8 ${ }^{+} \mathrm{T}$ cells, and myeloid dendritic cells, respectively. (H-J) No significant correlation between KPNA2 expression and infiltration of cytotoxic lymphocytes, neutrophils, and NK cells. (K) The significant negative correlation of KPNA2 with infiltration of endothelial cells. (L) No significant correlation between KPNA2 expression and infiltration of fibroblasts. The red node in Figure 5A represented KPNA2 protein, the black and blue nodes were the proteins which had liver-specific PPIs with KPNA2. The blue nodes also indicated the proteins significantly enriched in the KEGG pathways in Figure 5B. PPI, protein-protein interaction. Spearman correlation analysis was used and $p<0.05$ was considered significant. 


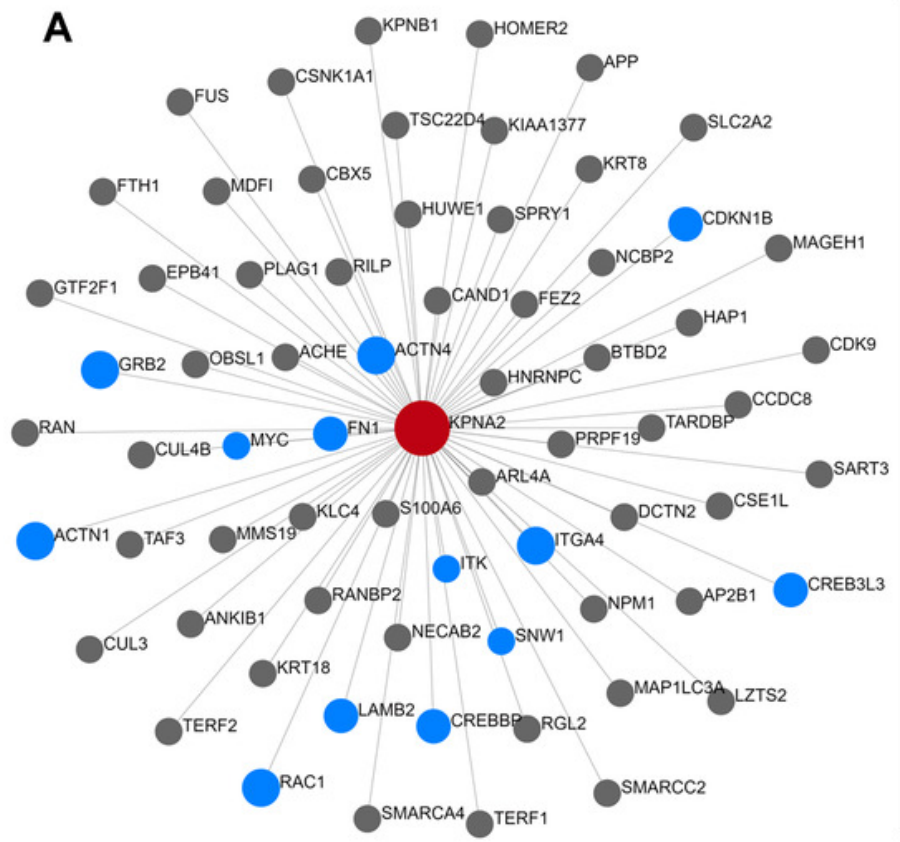

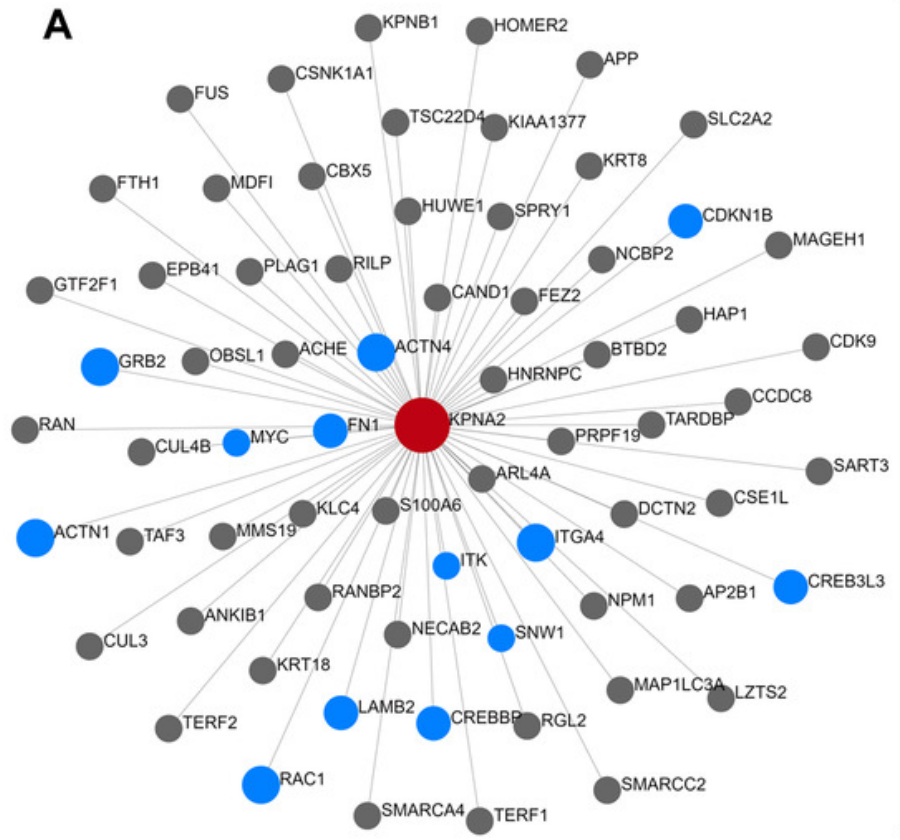

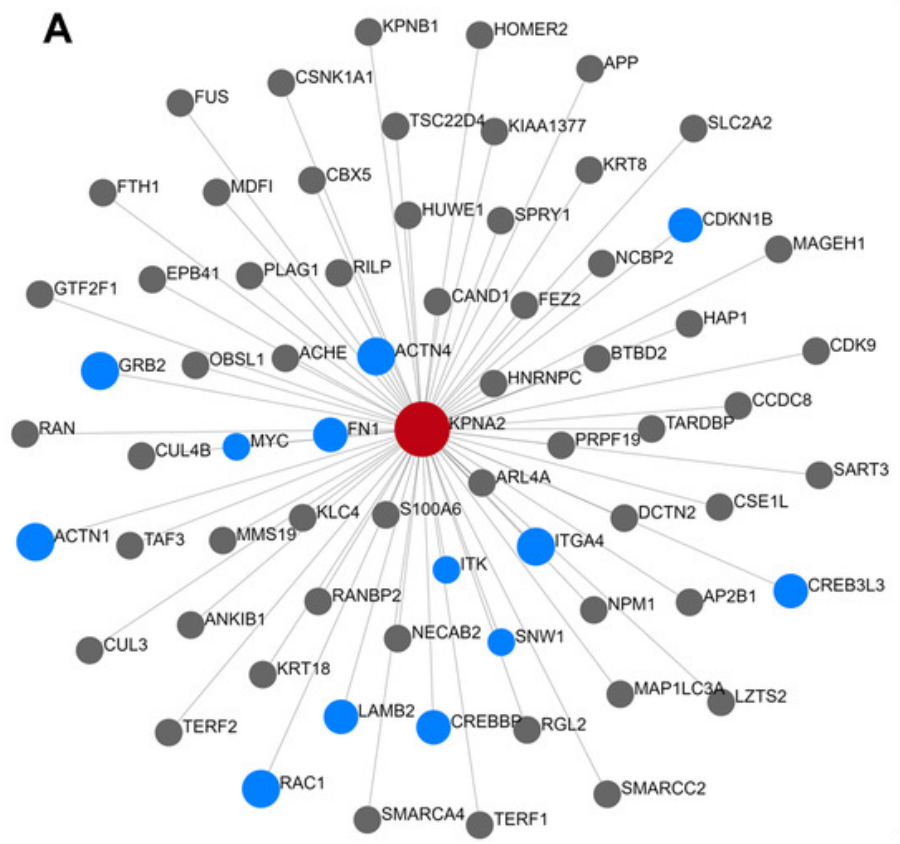

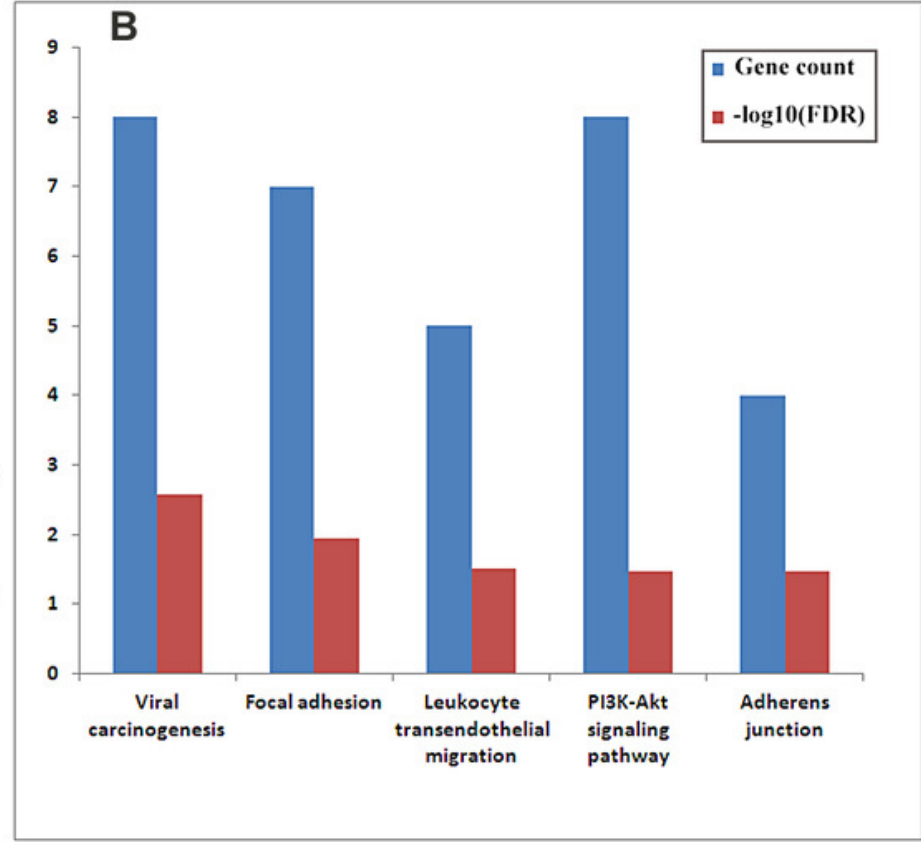

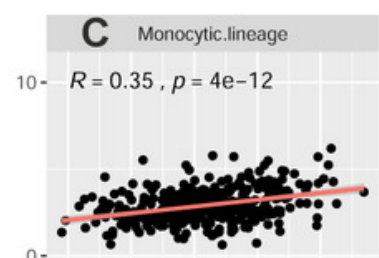

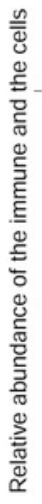

$$
\underset{R=0.23, p=1 \mathrm{e}-05}{\text { T.cells }}
$$

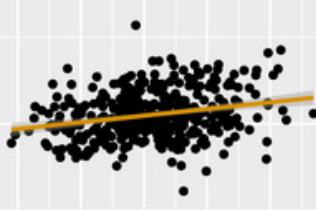

E B.lineage

$R=0.14, p=0.0071$

$R=0.14, p=0.006$
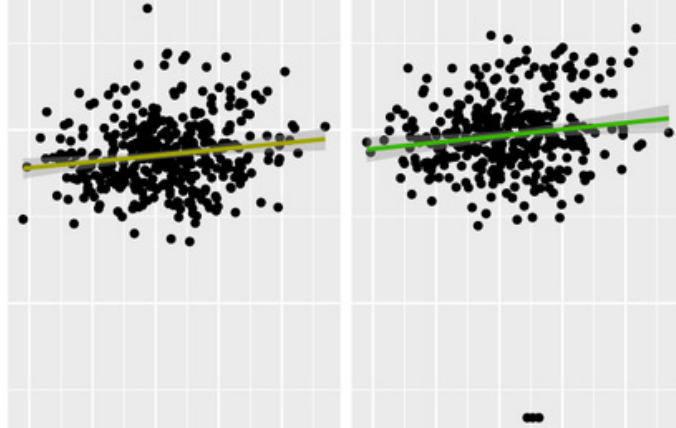

H cytotoxic.lymphocytes

$R=0.0083, p=0.87$

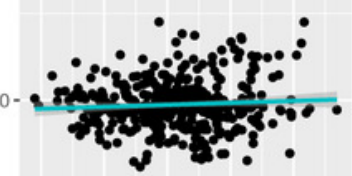

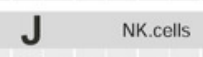

$R=-0.082, p=0.11$

$R=-0.084, p=0.11$

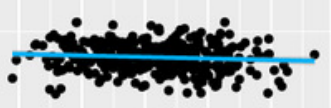

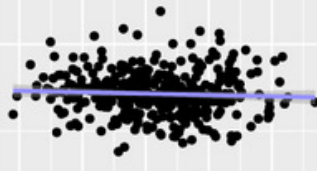

Myeloid.dendritic.cells

$R=0.13, p=0.014$

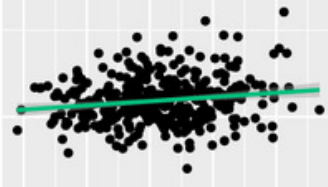

$-10-$

Relative expression of KPNA2 in HCC 


\section{Figure 6}

Prognostic value of KPNA2-correlated immune and stroma cells in HCC.

(A) The gender-, age-, and tumor stage- corrected prognostic value of KPNA2-correlated immune and stroma cells for HCC OS. (B) The gender-, age-, and tumor stage-corrected prognostic value of KPNA2-correlated immune and stroma cells for HCC DFS. OS, overall survival; DFS, disease-free survival. Cox regression analysis with ezcox package in $\mathrm{R}$ software was used for survival analysis and $p<0.05$ was considered significant. 


A
Variable
Monocytic.lineage
T.cells
CD8.T.cells
B.lineage
Myeloid.dendritic.cells
Endothelial.cells
B

Variable

Monocytic.lineage

T.cells

CD8.T.cells

B.lineage

Myeloid.dendritic.cells

Endothelial.cells

\section{Overall survival}

$\mathrm{HR}(95 \% \mathrm{Cl}) \quad$ Pvalue

$1.03(1.01-1.04) \quad 0.00347$

$1(0.956-1.05)$

0.927

$1(0.973-1.04)$

0.773

$0.987(0.944-1.03)$

0.571

$1.03(1.01-1.06)$

0.0176

$0.982(0.928-1.04)$

0.529

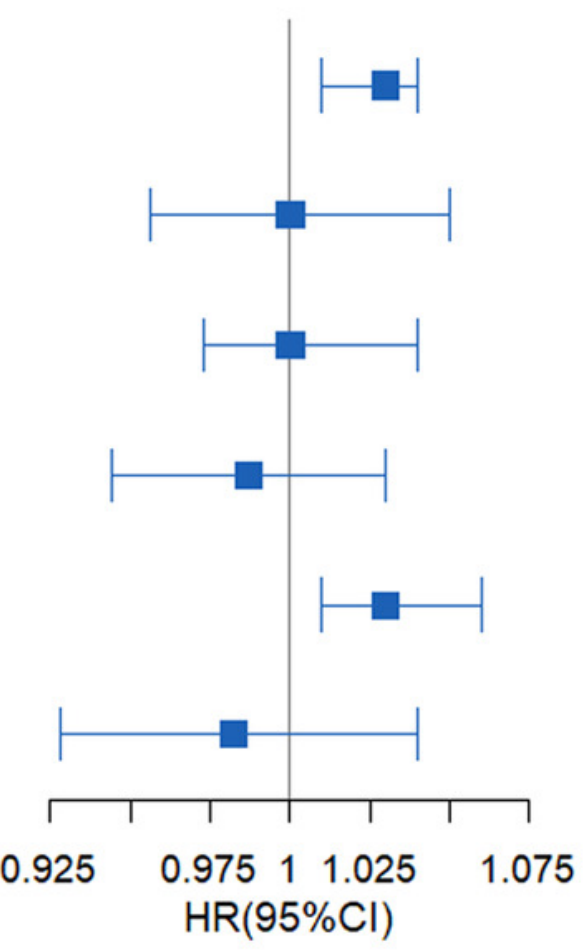

\section{Disease-free survival} $\mathrm{HR}(95 \% \mathrm{Cl}) \quad$ Pvalue

$1.01(0.991-1.03) \quad 0.354$

$1.01(0.951-1.06)$

0.843

$0.993(0.962-1.02)$

0.645

$1.01(0.993-1.03)$

0.209

$1.01(0.976-1.03)$

0.725

$0.968(0.924-1.01)$

0.179

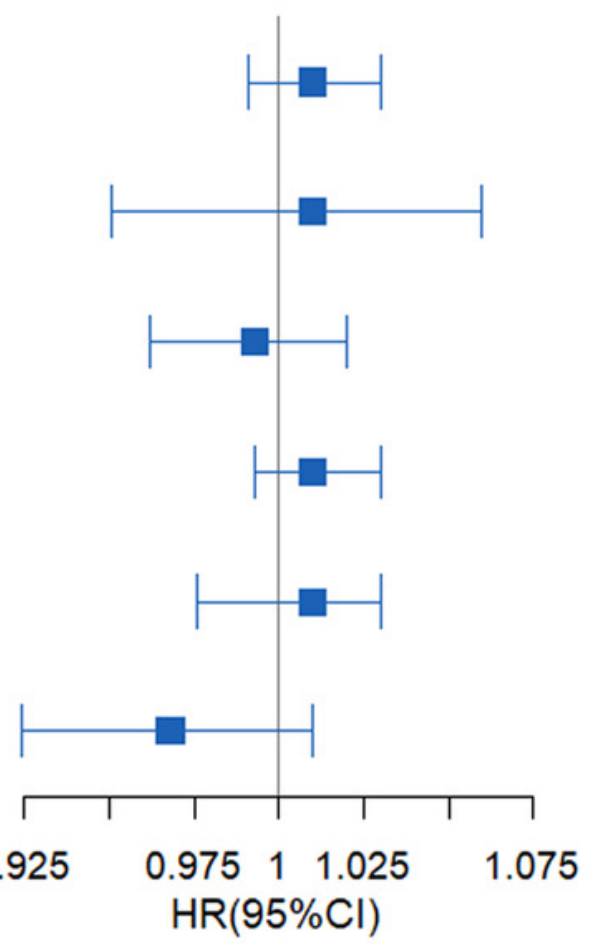


Figure 7

KPNA2 expression comparisons in immune cells between $\mathrm{HCC}$ samples and their paired normal livers.

One way ANOVA analysis was used for comparisons and $p<0.05$ was considered significant.
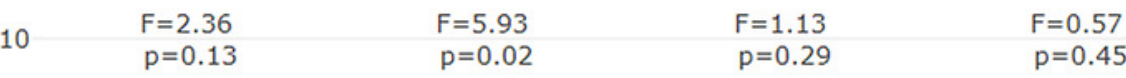

$F=16.58$
$p=5.58 e-5$
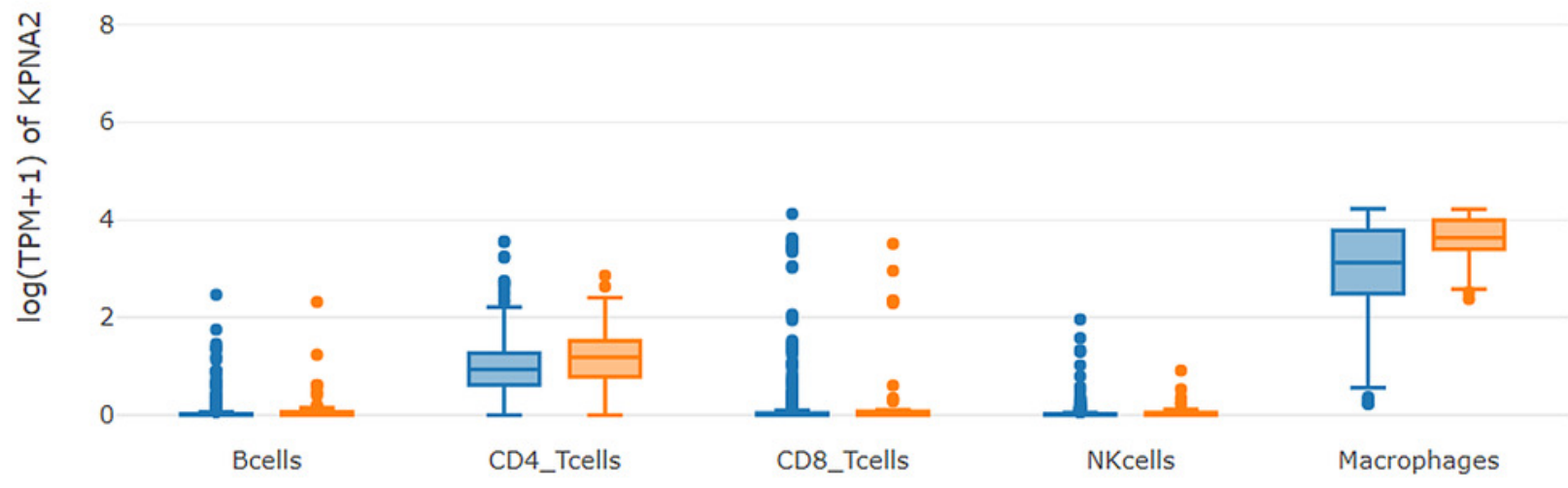


\section{Figure 8}

Associations between KPNA2 expression and fatty acid metabolism in liver and HCC.

(A) Significant decreases of KPNA2 expression in the obese livers with low-fat diet in contrast

to the controls. (B) Significant negative correlations between KPNA2 expression and the expression of the genes in KEGG fatty acid metabolism pathway. (C) Functional enrichment of KPNA2 negatively-correlated genes in KEGG fatty acid metabolism pathway in HCC. For (AB), GEO2R tool and Spearman correlation analysis was used respectively and $p<0.05$ was considered significant. For (C), the analysis was performed via Metascape ( https://metascape.org ) and the top 15 items were listed. 

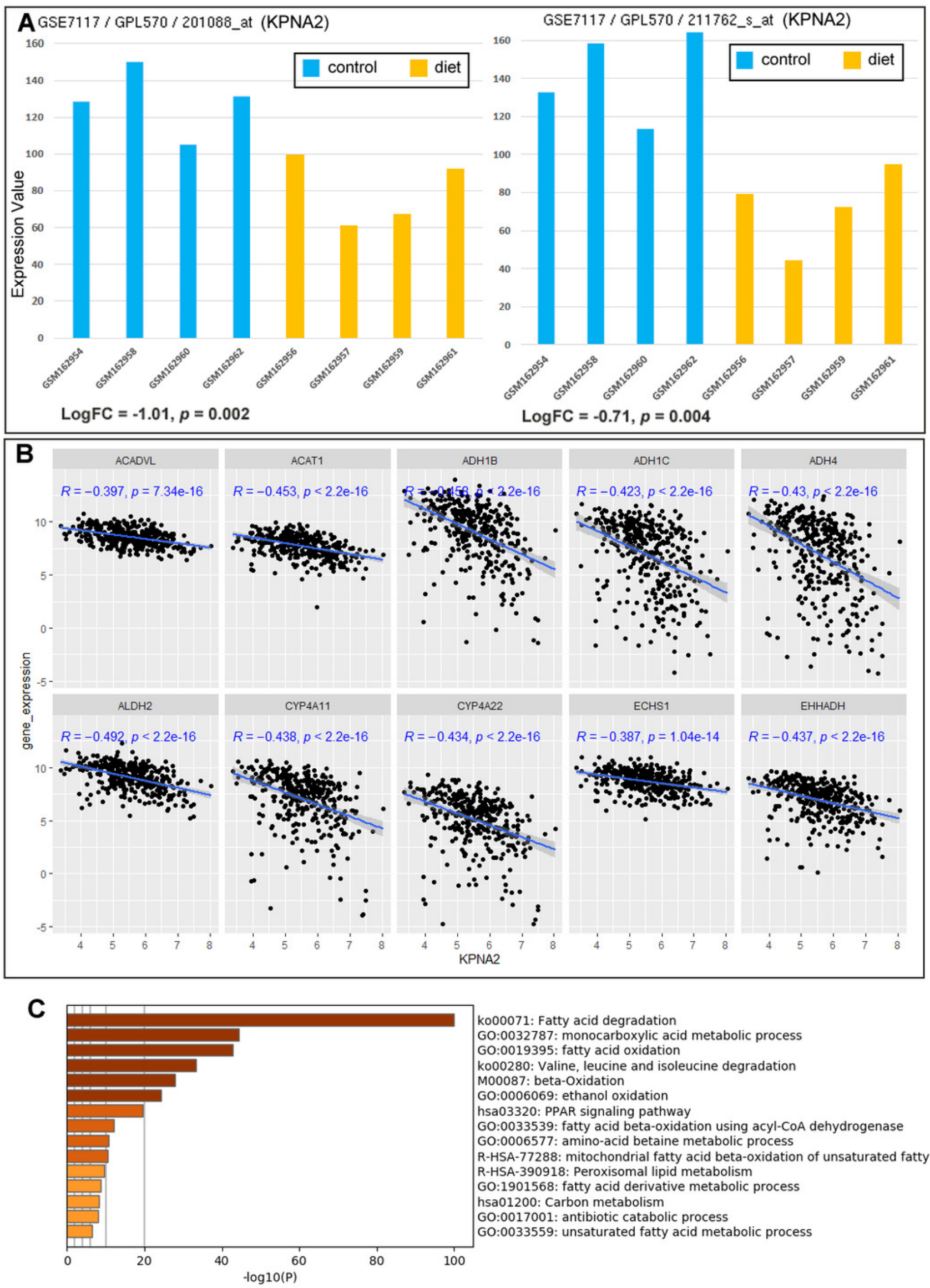

k000071: Fatty acid degradation

GO:0032787: monocarboxylic acid metabolic process GO:0019395: fatty acid oxidation

ko00280: Valine, leucine and isoleucine degradation

M00087: beta-Oxidation

GO:0006069: ethanol oxidation

hsa03320: PPAR signaling pathway

GO:0033539: fatty acid beta-oxidation using acyl-CoA dehydrogenase

GO:0006577: amino-acid betaine metabolic process

R-HSA-77288: mitochondrial fatty acid beta-oxidation of unsaturated fatty acids

R-HSA-390918: Peroxisomal lipid metabolism

GO:1901568: fatty acid derivative metabolic process

hsa01200: Carbon metabolism

GO:0017001: antibiotic catabolic process

GO:0033559: unsaturated fatty acid metabolic process 


\section{Figure 9}

Expression differences of KPNA2 various transcripts between $\mathrm{HCC}$ and the paired normal liver controls.

(A-B) ENST00000330459 and ENST00000537025 were higher expressed in HCC than the controls. (C-E) No significant expression differences of ENST00000579754, ENST00000584026, and ENST00000583269 between HCC and the controls. (F-G) ENST00000583392 and ENST00000582898 were higher expressed in HCC than the controls. Paired samples Wilcoxon test was used for expression comparisons and $p<0.05$ was considered significant.

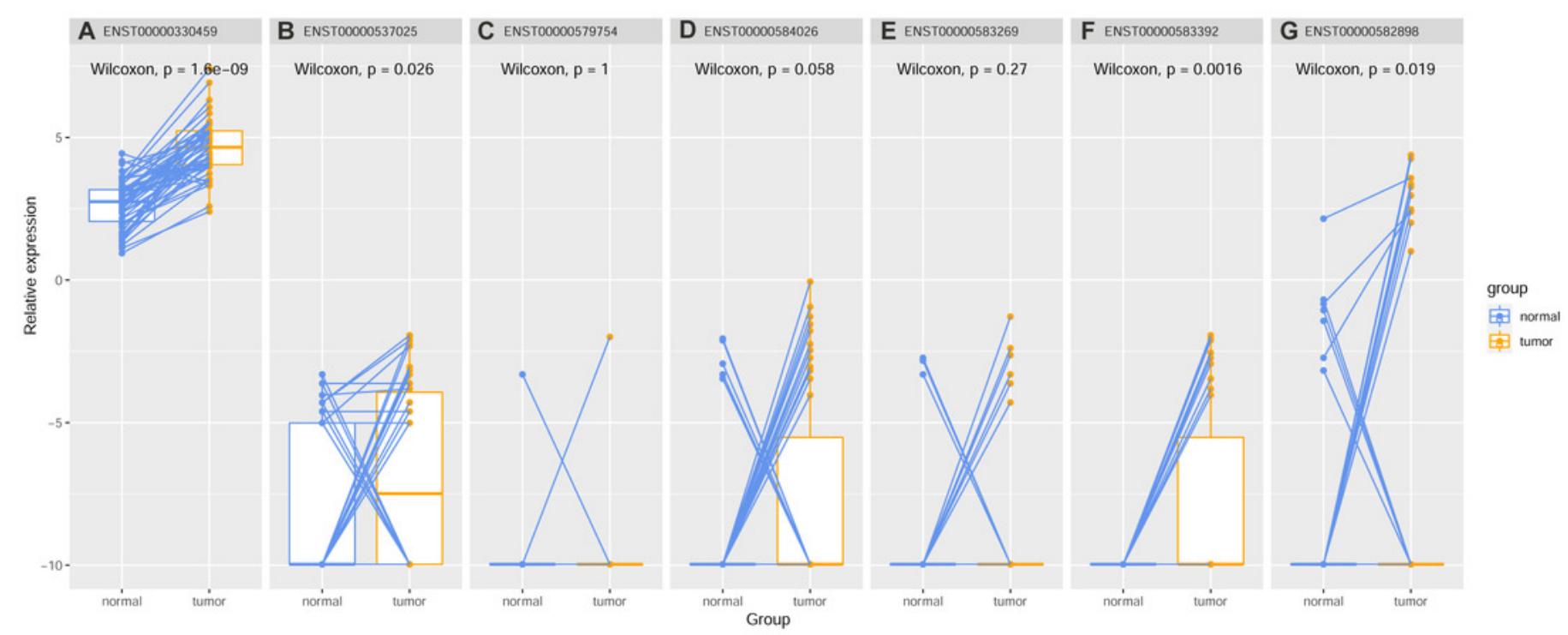




\section{Figure 10}

Prognostic value of KPNA2 transcripts in HCC.

(A) The significant prognostic value of ENST00000330459, ENST00000537025, and tumor stage for HCC OS. (B) The significant prognostic value of ENST00000330459 and tumor stage for HCC DFS. OS, overall survival; DFS, disease-free survival. Multivariable Cox regression analysis was used for survival analysis and $p<0.05$ was considered significant. 


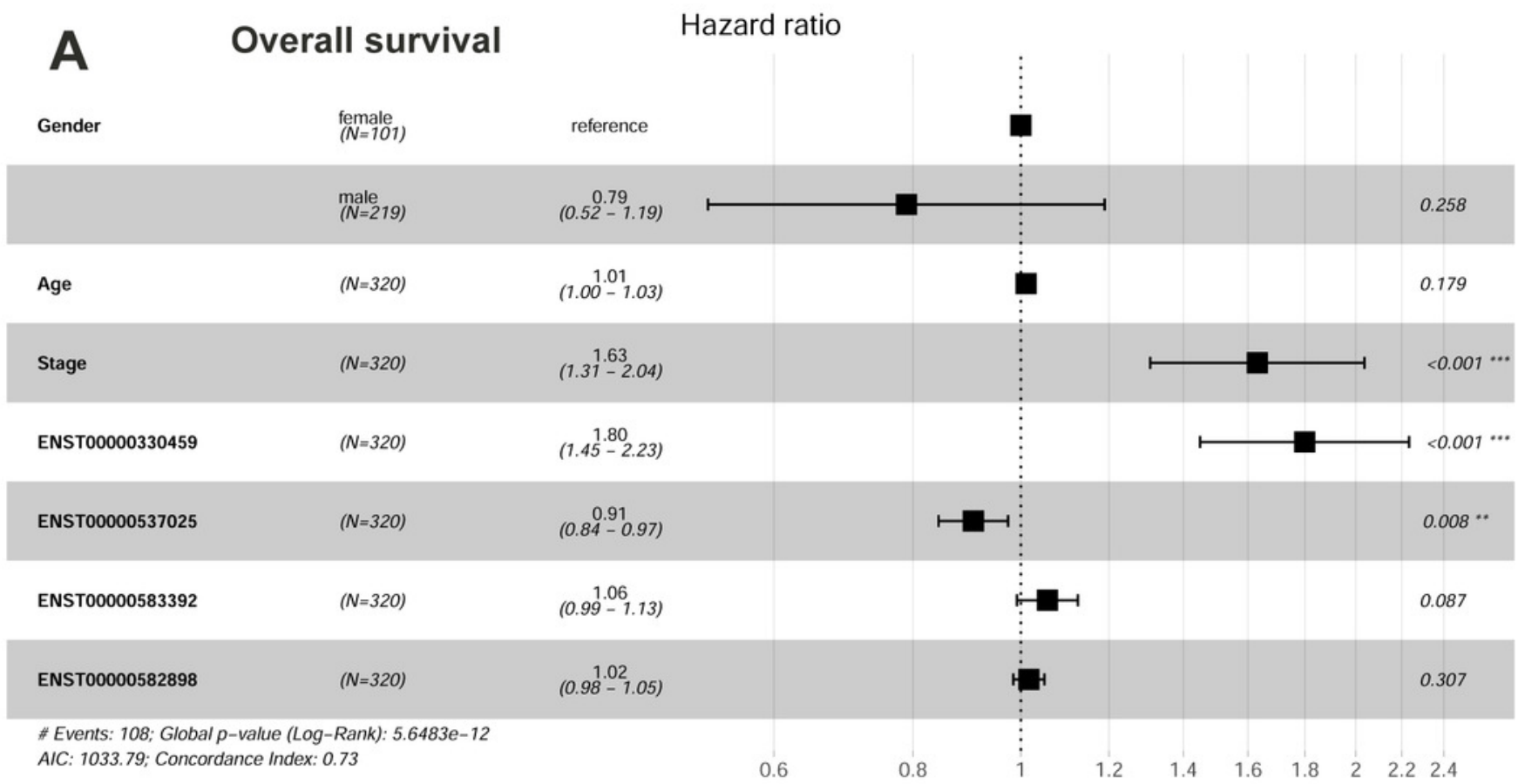

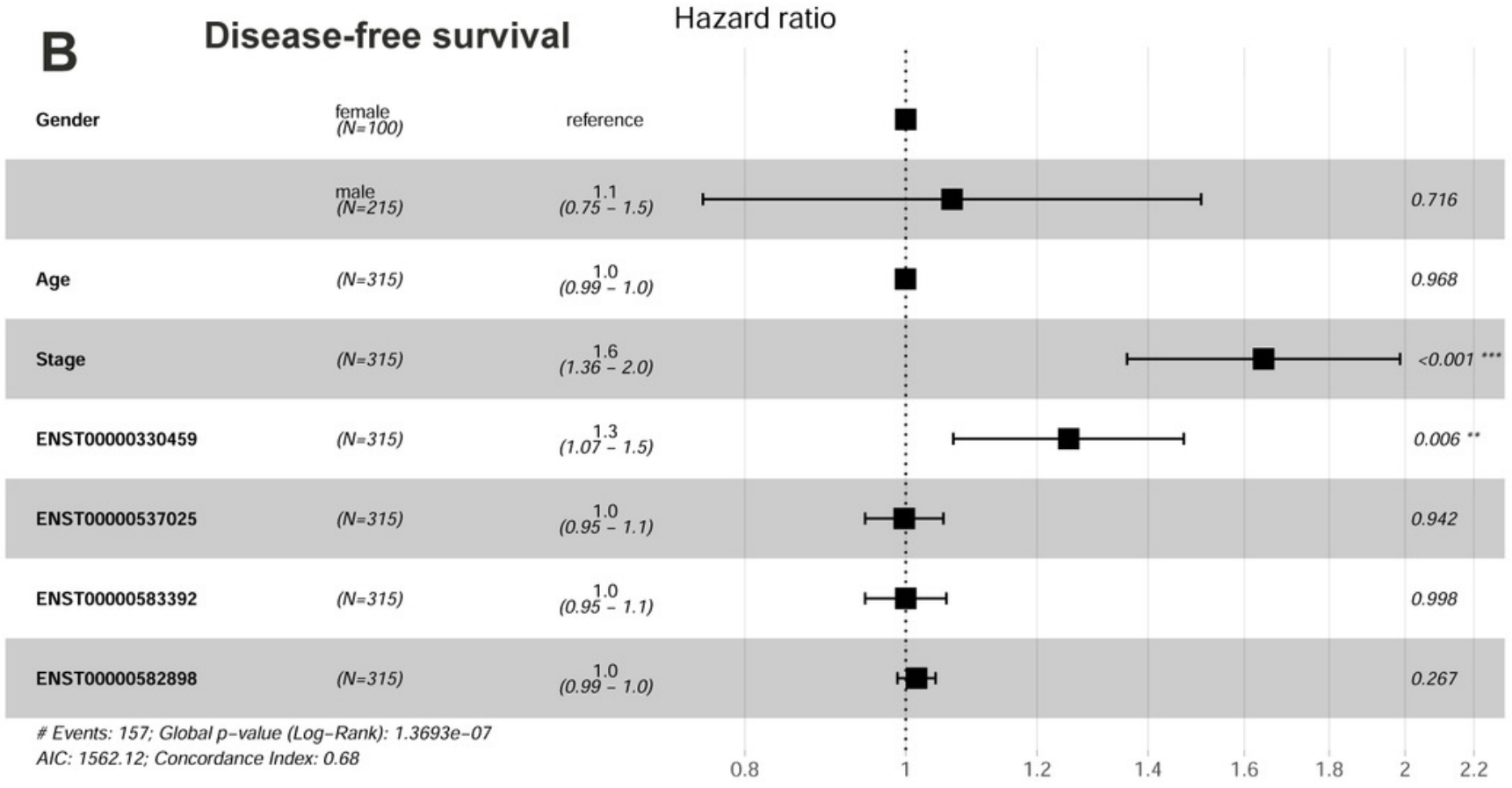




\section{Figure 11}

Immunohistochemical staining of KPNA2 in HCC and normal liver tissues from HPA database.

(A) With the antibody CAB015460, KPNA2 was positive in cytoplasm/membrane of normal liver cells with negative nuclear staining. (B) KPNA2 was positive in nuclei of HCC cells (with the antibody CAB015460) . (C) With the antibody HPA041270, KPNA2 was extensively stained in both cytoplasm/membrane and nuclei of normal liver cells. (D) The positive nuclear staining became more intensive in HCC tumor cells in comparison with (C) (with the antibody HPA041270). All the visual fields were extracted from same magnification and the scale plates were shown. 


\section{CAB015460}

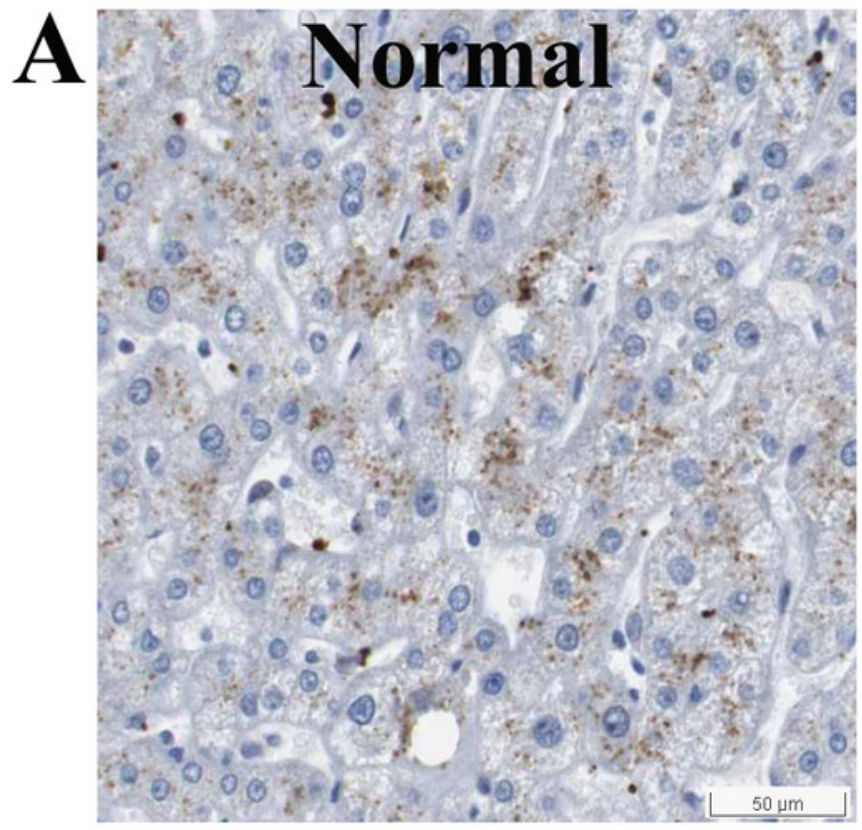

B

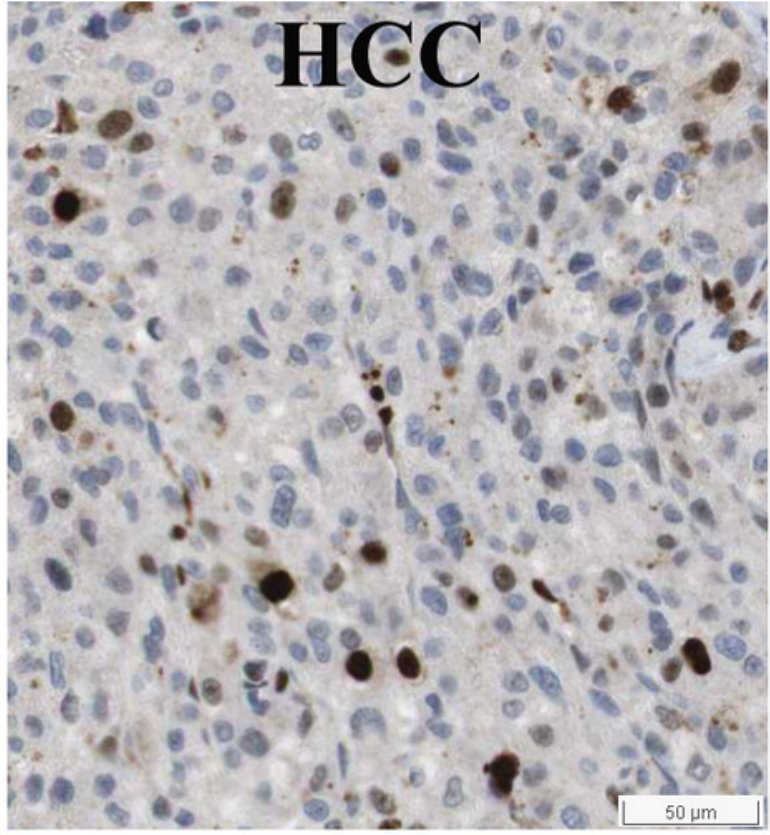

HPA041270

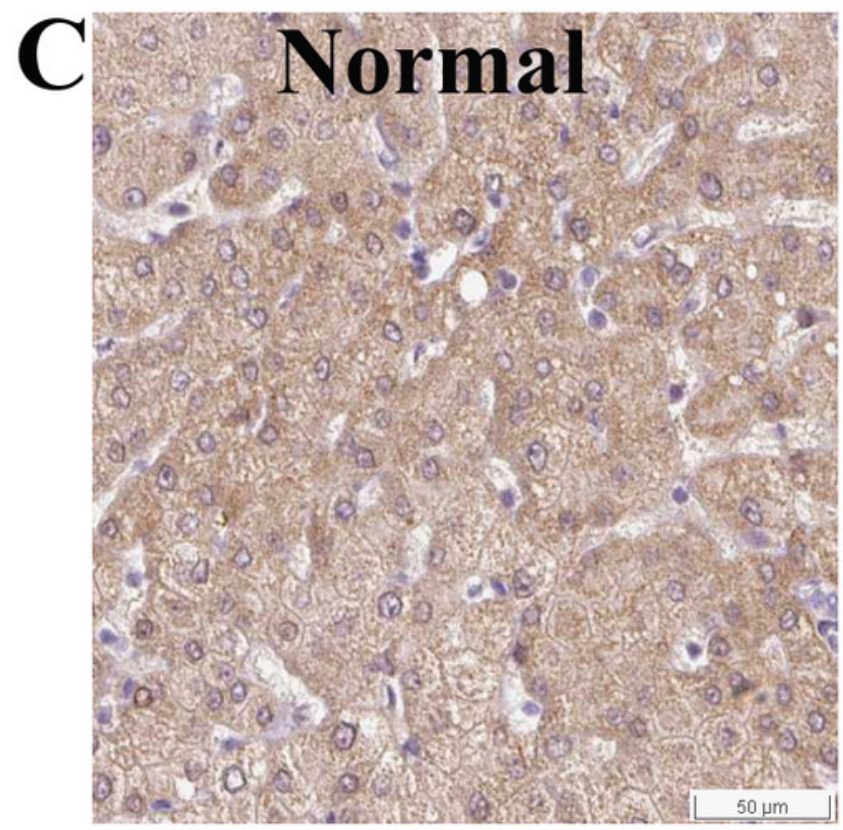

D

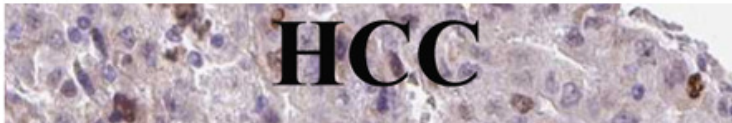

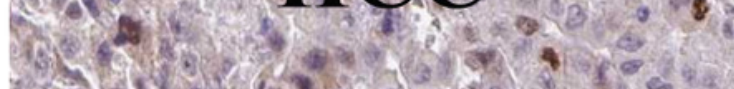

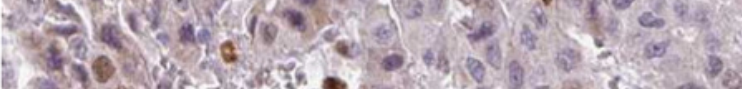

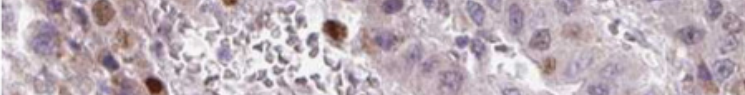

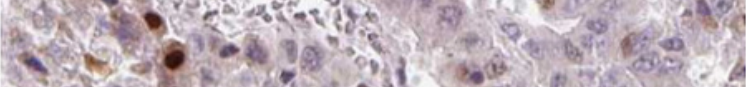

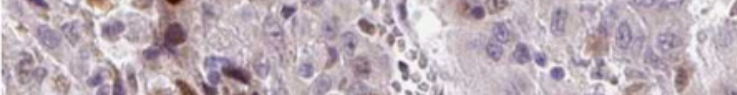

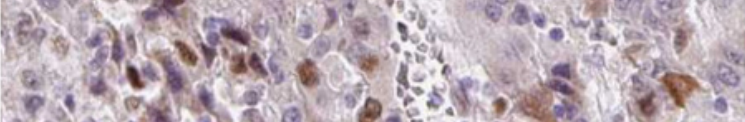

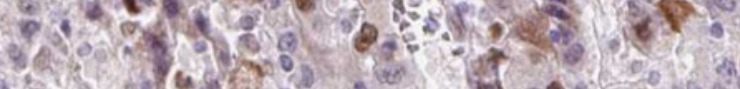

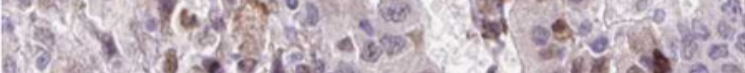
0.20 .

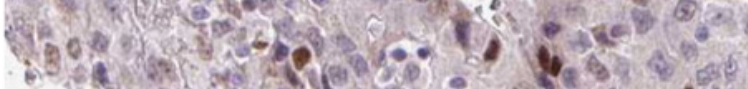

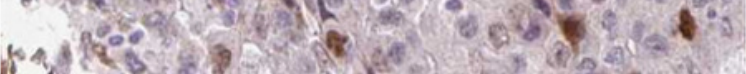

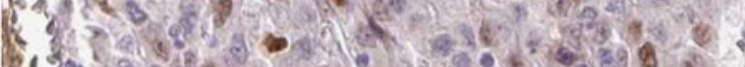
120. 


\section{Figure 12}

Plasma KPNA2 level and its discriminating power between HCC patients and healthy controls.

(A) Plasma KPNA2 concentration in HCC patients was lower than in healthy individuals by ELISA. (B) ROC curve analysis of plasma KPNA2 in discriminating HCC patients from healthy individuals. ROC, receiver operating characteristic; $p<0.05$ was considered to be statistically significant.
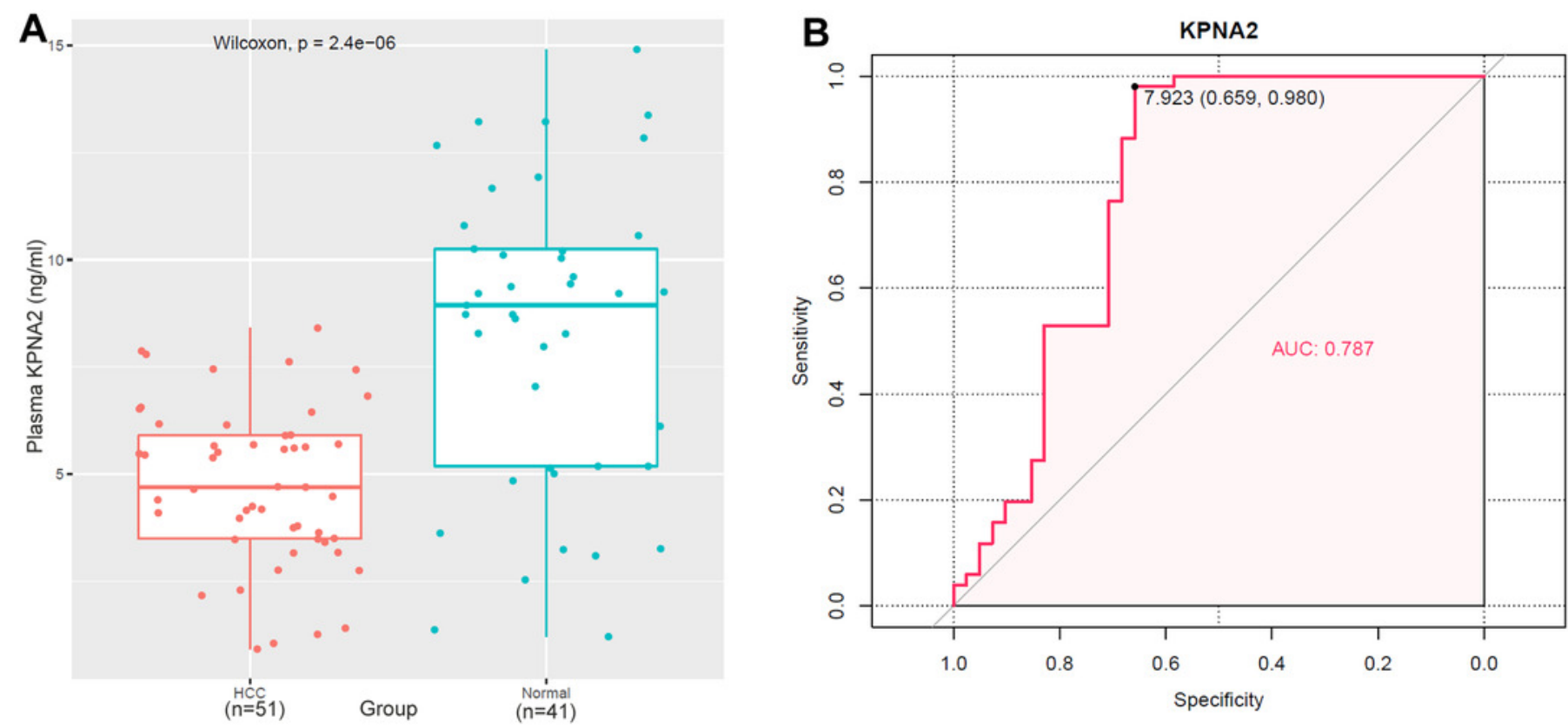